\title{
REVISIÓN E INTEGRACIÓN DEL CONOCIMIENTO de las Orchidaceae de Tabasco, México
}

\author{
Eliana Noguera-Savelli ${ }^{1,4}$ y William CetZal-Ix $x^{2,3}$ \\ ${ }^{1}$ Departamento de Conservación de la Biodiversidad, El Colegio de la Frontera Sur (ECOSUR), \\ San Cristóbal de Las Casas, Chiapas, México \\ ${ }^{2}$ El Colegio de la Frontera Sur (ECOSUR), Unidad Chetumal, Chetumal, Quintana Roo, México \\ ${ }^{3}$ Herbario CICY, Centro de Investigación Científica de Yucatán, Mérida, Yucatán, México \\ ${ }^{4}$ Autor para la correspondencia: eliananoguera@gmail.com
}

\begin{abstract}
Resumen: A la fecha, no existe un trabajo integrador y actualizado de la orquideoflora tabasqueña, lo anterior, a pesar de la existencia de diversos estudios florísticos donde se incluye a la familia y a la cantidad de material disponible en los herbarios locales. El objetivo de esta investigación fue recopilar, actualizar y ampliar el conocimiento de las orquídeas de Tabasco, mediante la realización de una lista con la información taxonómica actualizada, estado de conservación y la distribución geográfica de las especies. El trabajo incluyó para cada especie datos taxonómicos, hábito, comentarios taxonómicos, material de herbario, estado de conservación y la distribución geográfica en el estado y en sus áreas naturales protegidas. En Tabasco están presentes un total de 109 especies y una variedad, en 58 géneros. Se presentan doce nuevos registros para el estado: Anathallis yucatanensis, Cyrtopodium macrobulbon, Oeceoclades maculata, Maxillariella variabilis, Myrmecophila christinae var. christinae, Polystachya clavata, Prescottia stachyodes, Rhetinantha aciantha, Sarcoglottis assurgens, Stanhopea oculata, Stelis cobanensis y Trichosalpinx ciliaris. Seis de las especies presentes se encuentran en alguna categoría de riesgo, de acuerdo a la NOM-059-SEMARNAT-2010. Los municipios con una alta diversidad de especies son Teapa (66), Tacotalpa (55) y Huimanguillo (40). De acuerdo a los tipos de vegetación, en la selva mediana subperennifolia se encuentran 79 especies; en la selva alta perennifolia, 56 especies; en la selva baja inundable, 28 especies; y en la vegetación secundaria, 25 especies. La menor diversidad se registró en la selva baja subperennifolia y el manglar, con ocho especies cada una, seguido de la vegetación acuática con siete especies y el bosque mesófilo con cinco especies.
\end{abstract}

Palabras clave: estado de conservación, Orchidaceae, Tabasco, taxonomía.

\begin{abstract}
To date, there is no comprehensive and updated work on the orchids of Tabasco, despite the existence of several floristic studies that include Orchidaceae and citations of material available in local herbaria. The objective of this research was to collect, update, and expand our knowledge of the Orchidaceae of Tabasco, by compiling a list with all available taxonomic information, conservation status, and geographic distribution of species. The checklist includes a total of 109 species and one variety in 58 genera. Twelve taxa are new records for the state: Anathallis yucatanensis, Cyrtopodium macrobulbon, Oeceoclades maculata, Maxillariella variabilis, Myrmecophila christinae var. christinae, Polystachya clavata, Prescottia stachyodes, Rhetinantha aciantha, Sarcoglottis assurgens, Stanhopea oculata, Stelis cobanensis, and Trichosalpinx ciliaris. Six of the species are found in some risk category according to NOM-059-SEMARNAT-2010. The municipalities with high diversity of species are Teapa (66 species), Tacotalpa (55), and Huimanguillo (40). The vegetation types with the highest diversity are the evergreen tropical forest (79 species), the high evergreen forest (52), the lowland floodplain (28), and secondary vegetation (25). The lowest diversity was recorded in the lowland evergreen and mangrove, both with eight species, followed by the aquatic vegetation with seven species and cloud forest with five species.
\end{abstract}

Key words: conservation status, Orchidaceae, Tabasco, Taxonomy.

$\mathbf{L}$ a familia Orchidaceae cuenta con 788 géneros y 19,500 especies (Judd et al., 2008), constituye uno de los grupos de plantas con flores más grande y diverso. En México, se estima que existen 1,250 especies y 168 géneros de orquí- deas (Soto et al., 2007; Salazar, 2013), muy probablemente esta cifra pueda variar y elevarse a 1,300-1,400 especies, en la medida en que se hace más detallado el conocimiento de la orquideoflora del país. En este sentido, el conocimiento 
de las orquídeas de Tabasco es escaso, se encuentra disperso en diferentes publicaciones y está desactualizado taxonómicamente. Entre los trabajos dedicados a las Orchidaceae de Tabasco o que incluyen entre sus listados a especies de esta familia de plantas, se encuentran: Cowan (1983), Alderete y Cappello (1988), Soto (1988), Alderete (1992), RamónJiménez (1992), López-Hernández (1994), Hanan-Alipi (1997), Espejo y López-Ferrari (1997, 1998), Cruz-Hernández (2000), Carnevali et al. (2001), Pérez et al. (2005), Novelo y Ramos (2005), Díaz-Jiménez (2007) y Carnevali et al. (2010). Los anteriores, con estimaciones variables en cuanto al número de especies, al área y al ecosistema muestreado para el estado y con diferentes clasificaciones sistemáticas para los géneros y especies.

Una de las primeras aportaciones al conocimiento de las orquídeas de Tabasco y de las más ampliamente citadas corresponde la de Cowan (1983), publicación que ha representado la base para la gran mayoría de todos los trabajos subsiguientes, además de ser la principal referencia en cuanto a las determinaciones y los ejemplares de herbario citados. Otro trabajo dedicado a orquídeas del estado lo constituye el de Alderete y Cappello (1988), quienes resaltaron como aporte de su investigación la inclusión de 43 especies no consideradas en trabajos previos. Posteriormente, Cruz-Hernández (2000) registró 145 especies distribuidas en 51 géneros de orquídeas, y mencionó datos en torno a la distribución de las especies y su potencial como plantas ornamentales. En un estudio en el que se trata, en general, la biodiversidad de Tabasco, Pérez et al. (2005) reconocieron 105 especies de orquídeas, y aumentó considerablemente el número registrado previamente para el estado por otros autores, a excepción de Cruz-Hernández (2000). Algunos de estos trabajos carecen de la cita de ejemplares de herbario que respalden la identificación de los especímenes y que confirmen su presencia en el estado, lo que consittuye una limitación para la veracidad de la identificación de las especies registradas; por otra parte, se incluyen a especies esperadas en el total de taxa presentes.

Hasta la fecha no existe un trabajo integrador y actualizado de la orquideoflora tabasqueña, por lo que surge esta investigación con el objetivo de contribuir al conocimiento taxonómico, la distribución y conservación de las Orchidaceae del estado de Tabasco, México.

\section{Materiales y métodos}

Área de estudio. Tabasco políticamente se encuentra dividido en 17 municipios que abarcan una extensión territorial de $24,731 \mathrm{~km}^{2}$ y ocupan el $1.3 \%$ de la superficie del país. Velázquez-Villegas (1994) basado en características morfológicas, hidrográficas y socioeconómicas, dividió el estado en cuatro regiones: Región de la Chontalpa, que abarca el $35 \%$ de la extensión del estado y está conformada por los municipios Huimanguillo (H), Cárdenas (C), Cunduacán
$(\mathrm{Cu})$, Paraíso (P), Nacajuca (N), Jalpa de Méndez (JM) y Comalcalco (Co); la región de los ríos, influida principalmente por los ríos Usumacinta y Grijalva, ocupa el $42 \%$ del territorio tabasqueño y comprende los municipios de Tenosique (Teno), Emiliano Zapata (EZ), Balancán (B), Jonuta (Jo) y Centla (Ce); mientras que Macuspana (M), Teapa (Te), Tacotalpa (Ta) y Jalapa (J) forman la región de la Sierra, que representa el 16\% del territorio estatal; por último, la región del Centro (Cen) está conformada por el municipio del mismo nombre y ocupa el 7\% del territorio Tabasqueño. El 96\% del territorio de Tabasco está conformado por la llanura costera del Golfo sur, dominada por humedales y, aunque su extensión es mayor a la de la zona costera, ambas delimitaciones espaciales se sobreponen. El restante 4\% del territorio estatal se encuentra en las porciones montañosas que forman parte de las sierras norte de Chiapas y las sierras bajas del Petén, en los municipios de Huimanguillo, Teapa, Tacotalpa y Tenosique (INEGI, 2007).

Biogeográficamente, Tabasco forma parte de la provincia del Golfo de México, que incluye a los estados de Veracruz, Tamaulipas, San Luis Potosí, Hidalgo, Puebla, Oaxaca, Chiapas y Campeche, Belice y el norte de Guatemala (Morrone, 2001).

Revisión y determinación taxonómica. Se consultaron las colecciones de los herbarios CICY, CSAT y UJAT. Asimismo, se revisaron las bases de datos e imágenes de material de los herbarios MEXU, MO y XAL. Además, se revisaron imágenes de los herbarios AMES, F, G, K, LL, MICH, NY, P, US y W (Acrónimos de acuerdo a Holmgren et al., 1990). También se consultó literatura local, regional y nacional (tesis de licenciatura, floras y listados florísticos), que incluyeran especies de orquídeas de Tabasco. La taxonomía y nomenclatura a nivel genérico se basó en Genera Orchidacearum (Chase, 2009), con excepción de los géneros Cohniella y Lophiaris que son discutidos en la propuesta de Carnevali et al. (2013). En los casos disponibles para cada especie se ubicó los duplicados de los especímenes de los herbarios CSAT y UJAT, depositados en AMO, CAS, CORU, ENCB, MEXU, MO y XAL. Adicional a la revisión de herbarios, dentro del marco de las actividades del proyecto "Gestión y estrategias de manejo sustentable para el desarrollo regional en la cuenca hidrográfica transfronteriza Grijalva", se realizaron muestreos en la porción montañosa de Tacotalpa, en fragmentos de selva mediana subperennifolia, en las localidades: Cerro Los Aguacates, Cerro de La Campana, Grutas de Kolem Chen, Grutas de San Antonio, en los ejidos Cuviac, Oxolotán, La Pila y Tomás Garrido. Los ejemplares de respaldo se depositaron en los herbarios $\mathrm{CH}$ y CICY. Los ejemplares vivos se mantienen en reguardo en los viveros del ECOSUR, Unidad San Cristóbal de Las Casas, Chiapas.

En el listado, los taxa se encuentran organizados en orden alfabético, en los casos necesarios se indicó para cada 
especie las identificaciones previas que fueron asignadas por diversos autores en la literatura de la flora de Tabasco. Además, se incluyó para cada taxa los municipios donde se encuentran registrados el tipo de vegetación, el hábito, los colectores y el herbario donde se encuentra depositado el espécimen, algunos comentarios taxonómicos y el estado de conservación de acuerdo a la NOM-059-SEMARNAT (SEMARNAT, 2010). Las especies registradas para Tabasco que carecen de un espécimen de herbario de respaldo en los diversos estudios consultados fueron excluidas del listado actualizado (Apéndice 1).

En cuanto al mapa de distribución de las especies en áreas naturales protegidas estatales y municipales (ANP), este se elaboró con base en los datos geográficos de las localidades citadas en las etiquetas de los especímenes de herbario y los citados en la literatura (Apéndice 1), para ser proyectado en un mapa base de ANP (Bezaury-Creel et al., 2007), mediante el programa ArcView 3.2 (ESRI, 1999). Los tipos de vegetación que se describen, donde crecen las especies, fueron citados explícitamente de las etiquetas de los especímenes de herbario, en algunas casos se tuvieron que inferir a partir de mapas de vegetación del sistema de clasificación del Instituto Nacional de Estadística y Geografía (INEGI, 2011). La mayor parte de las localidades se representaron gráficamente, algunas localidades redundantes no se registraron para facilitar el entendimiento del mapa.

\section{Resultados}

Diversidad de especies y actualizaciones taxonómicas. Se registraron 58 géneros, 109 especies y una variedad (Cuadro 1, Apéndice 1, Figuras 1-2). Los géneros con mayor número de especies son Epidendrum (15), Lophiaris (5), Prosthechea (5), Habenaria (4), Specklinia (4) y Vanilla (4). Estos seis géneros representan el 33\% (37) de los taxa presentes en Tabasco. En lo que respecta al hábito, el $83 \%$ de las especies son epífitas, $14 \%$ terrestres y $3 \%$ hemiepífitas.

$\mathrm{Al}$ analizar el cuadro 1 se puede observar que para cada trabajo citado, aproximadamente solo la mitad de las especies registradas corresponde con el nombre actualmente aceptado, así se tiene p. ej., Alderete y Cappello (1988), de los 43 taxa registrados, 20 (47\%) representan nombres aceptados, diez especies requirieron de actualización taxonómica y 11 nombres corresponden a identificaciones incorrectas; para este trabajo se realizaron 14 actualizaciones con base en la nuevas clasificaciones taxonómicas de la familia. Ramón-Jiménez (1992), de los 48 taxa registrados, 23 (48\%) representa nombres aceptados, y ocho corresponden a identificaciones incorrectas; se realizaron 15 actualizaciones a los nombres considerados en este trabajo. En Pérez et al. (2005), de los 105 taxa registrados, sólo 51 (49\%) representan nombres aceptados, 24 de las especies requirieron de actualización taxonómica y 17 corresponden a taxa excluidos; nueve son identificaciones incorrectas y 19 especies fueron registradas como esperadas. Este patrón, en el que aproximadamente la mitad de los nombres registrados están aceptados, y la actualización taxonómica y las determinaciones incorrectas corresponden a la otra mitad de las especies incluidas se repite en la gran mayoría de los estudios citados.

Nuevos registros. Se aportan 12 nuevos registros para las orquídeas de Tabasco: Anathallis yucatanensis, Cyrtopodium macrobulbon, Maxillariella variabilis, Myrmecophila christinae var. christinae, Oeceoclades maculata, Polysta-

Cuadro 1. Comparación de taxa de orquídeas incluidas en estudios de país, regionales y locales por diferentes autores para Tabasco. DetGen = Determinación hasta género. T-excl $=$ Taxones excluidos. T-esp $=$ Taxones esperados. Det-Inc $=$ Determinación incorrecta. $\mathrm{N}$-Actu $=\mathrm{Nombres}$ actualizados. N-Acep $=$ Nombres aceptados en este estudio.

\begin{tabular}{|c|c|c|c|c|c|c|c|c|c|}
\hline Autores & Taxa & Spp. & Var. & DetGen & T-excl & T-esp & Det-Inc & N-Actu & N-Acep \\
\hline Cowan (1983) & 46 & 39 & 1 & 8 & 7 & - & 21 & 11 & 10 \\
\hline Alderete y Cappello (1988) & 43 & 41 & - & 4 & 2 & - & 11 & 10 & 20 \\
\hline Soto (1988) & 43 & 38 & - & - & 4 & - & 7 & 14 & 18 \\
\hline Alderete (1992) & 83 & 58 & - & 1 & 26 & - & 28 & 17 & 29 \\
\hline Ramón-Jiménez (1992) & 48 & 45 & - & - & 3 & - & 8 & 15 & 23 \\
\hline López-Hernández (1994) & 21 & 18 & - & 1 & 3 & - & 6 & 6 & 8 \\
\hline Hanan-Alipi (1997) & 58 & 55 & - & 1 & 3 & - & 5 & 17 & 33 \\
\hline Espejo y López-Ferrari (1997-1998) & 48 & 42 & - & - & 6 & - & 3 & 18 & 22 \\
\hline Cruz-Hernández (2000) & 145 & 100 & - & 2 & 45 & - & 26 & 36 & 60 \\
\hline Carnevali et al. (2001) & 18 & 17 & - & - & 1 & - & - & 3 & 14 \\
\hline Pérez et al. (2005) & 105 & 79 & - & - & 17 & 19 & 9 & 24 & 51 \\
\hline Novelo y Ramos (2005) & 23 & 22 & - & - & 1 & - & 1 & 7 & 14 \\
\hline Carnevali et al. (2010) & 48 & 44 & 1 & - & 3 & - & - & 2 & 43 \\
\hline Este estudio & 110 & 109 & 1 & - & - & - & - & - & - \\
\hline
\end{tabular}



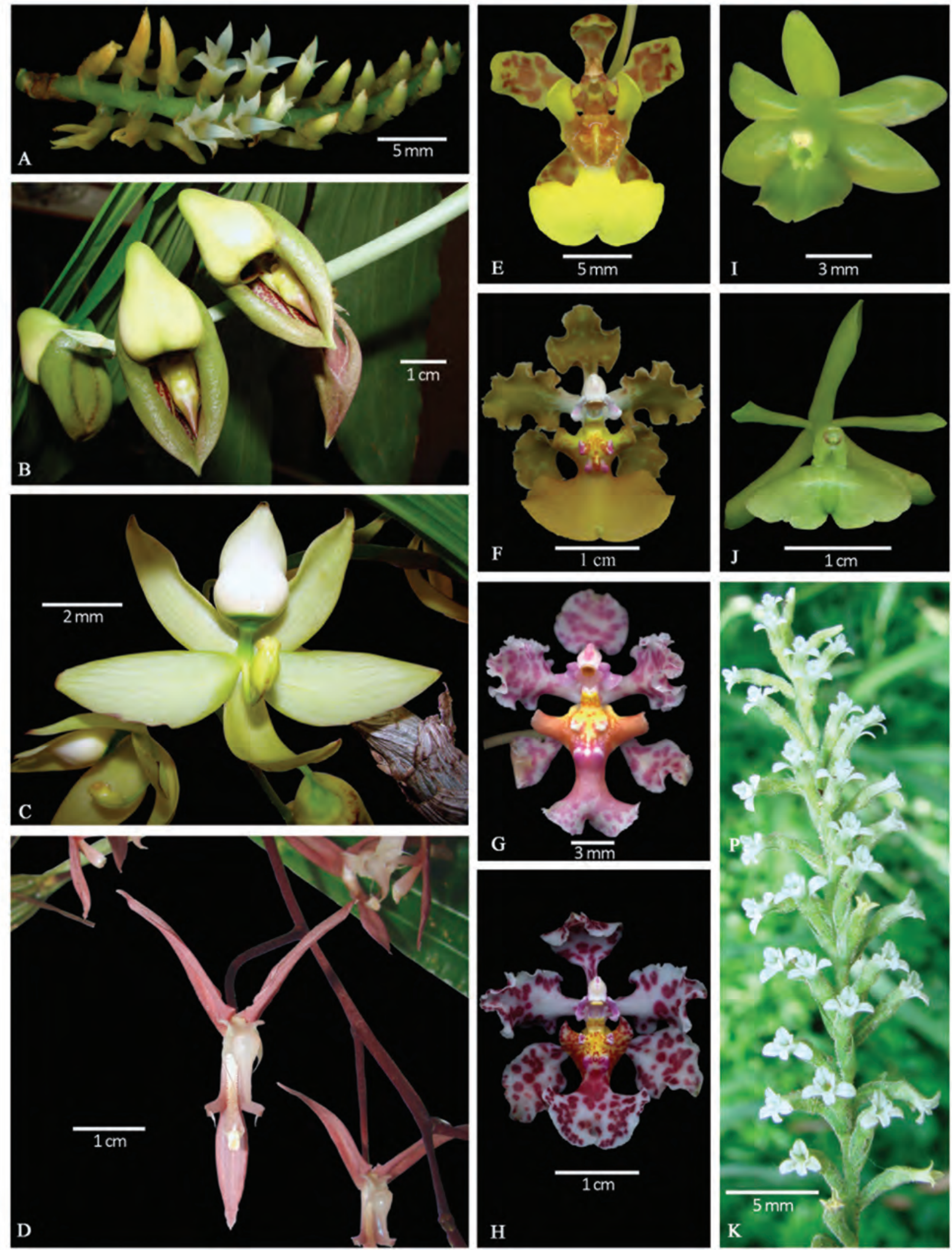

Figura. 1. Orquídeas de Tabasco. A) Campylocentrum micranthum. B) Catasetum integerrimum. C) Cycnoches ventricosum. D) Gongora unicolor. E) Cohniella ascendens. F) Lophiaris lurida. G) Lophiaris oerstedii. H) Lophiaris teaboana. I) Epidendrum cardiophorum. J) Epidendrum chlorocorymbos. K) Beloglottis costaricensis. 

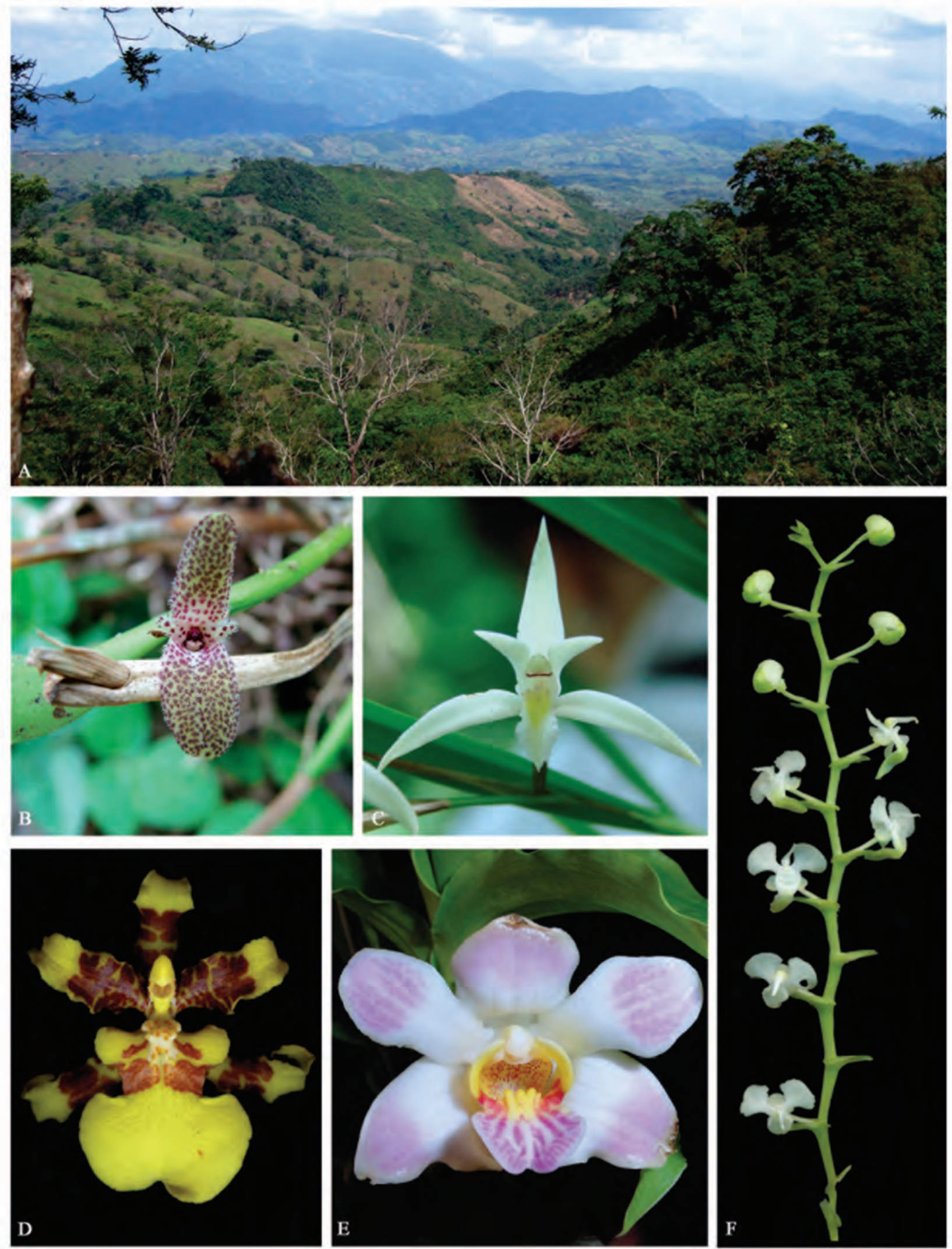

Figura. 2. Paisaje y orquídeas de Tabasco. A) Tacotalpa, Ejido Cuviac. B) Restrepiella ophiocephala. C) Nidema boothii. D) Oncidium sphacelatum. E) Chysis liminghei. F) Ornithocephalus inflexus. 


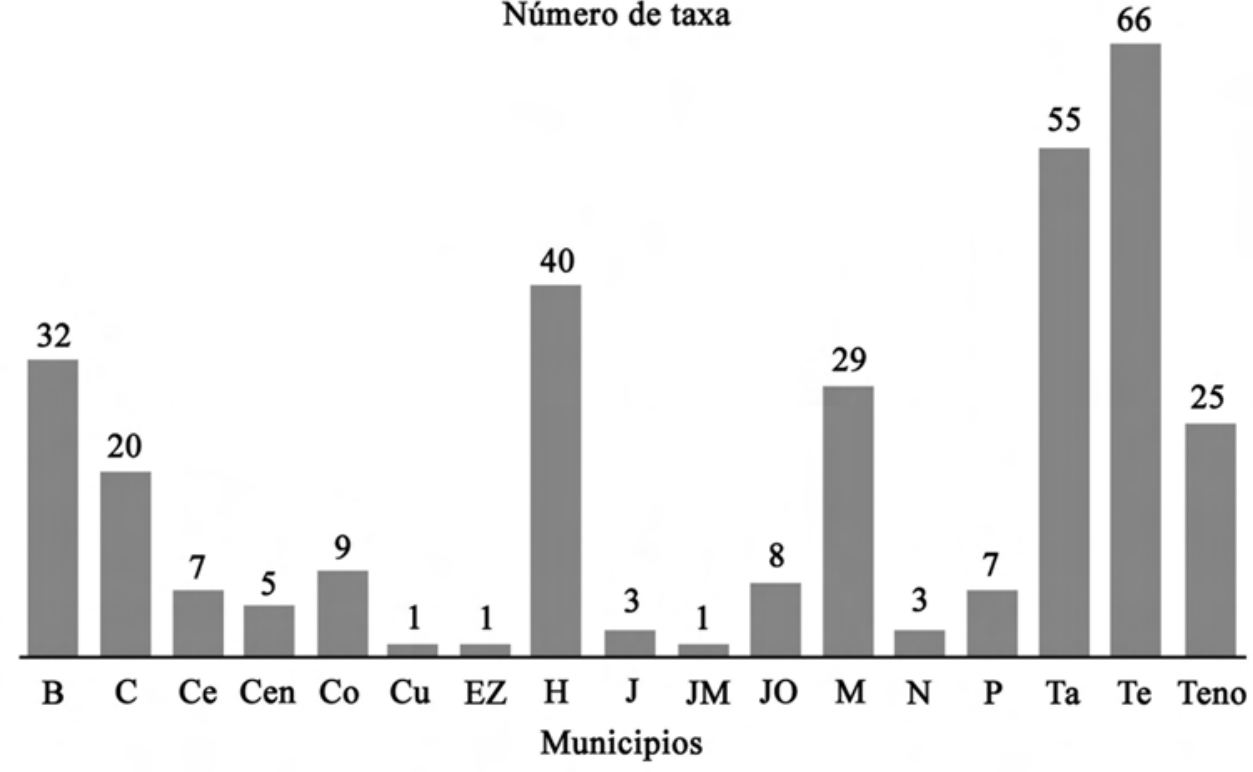

Figura 3. Número de taxa por municipios en Tabasco. Abreviaciones de los municipios: Balancán (B), Cárdenas (C), Centla (Ce), Centro (Cen), Comalcalco (Co), Cunduacán (Cu), Emiliano Zapata (EZ), Huimanguillo (H), Jalapa (J), Jalpa de Méndez (JM), Jonuta (Jo), Macuspana (M), Nacajuca (N), Paraíso (P), Tacotalpa (Ta), Teapa (Te) y Tenosique (Teno).

chya clavata, Prescottia stachyodes, Rhetinantha aciantha, Sarcoglottis assurgens, Stanhopea oculata, Stelis cobanensis y Trichosalpinx ciliaris. De los cuales, ocho están basados en colecciones de herbario y las restantes en colecciones realizadas por los autores (Apéndice 1). También con base en las colecciones del herbario UJAT se confirmó la presencia de Coelia triptera.

Distribución y Abundancia. Los municipios con mayor diversidad de especies son Teapa (66), Tacotalpa (55) y Huimanguillo (40); mientras que en Cunduacán, Emiliano Zapata y Jalpa de Mendéz se registró únicamente una especie (Figura 3). En Teapa se encuentra el 60\% y en Tacotalpa el 50\% de la orquideoflora de Tabasco, ambos municipios con vegetación primordialmente de selva alta perennifolia y selva mediana subperennifolia.

De acuerdo a los tipos de vegetación, en la selva mediana subperennifolia están presentes 79 especies, las más frecuentes son: Camaridium pulchrum, Cohniella ascendens, Dimerandra emarginata, Epidendrum chlorocorymbos, Maxillariella tenuifolia, Oncidium sphacelatum, Prosthechea pygmaea, P. radiata y Specklinia grobyi. Como terrestres y/o rupícolas fueron frecuentes Oeceoclades maculata y Prescottia stachyodes. En la selva alta perennifolia están presentes 56 especies, de las cuales son frecuentes como epífitas: Camaridium pulchrum, Cohniella ascendens, Epidendrum ramosum, Lophiaris lurida, Nemaconia striata, Nidema boothii, Oncidium sphacelatum, Prosthechea cochleata y Specklinia grobyi. En el caso de las terrestres, la más frecuente fue Sarcoglottis sceptrodes. Fue común observar individuos de Lophiaris lurida y Cohniella ascen- dens creciendo en simpatría en la selva alta perennifolia, en la zona de Teapa y Tacotalpa.

En la selva baja inundable están presentes 28 especies y en la vegetación secundaria 25 especies, en esta última es

\section{Número de taxa}

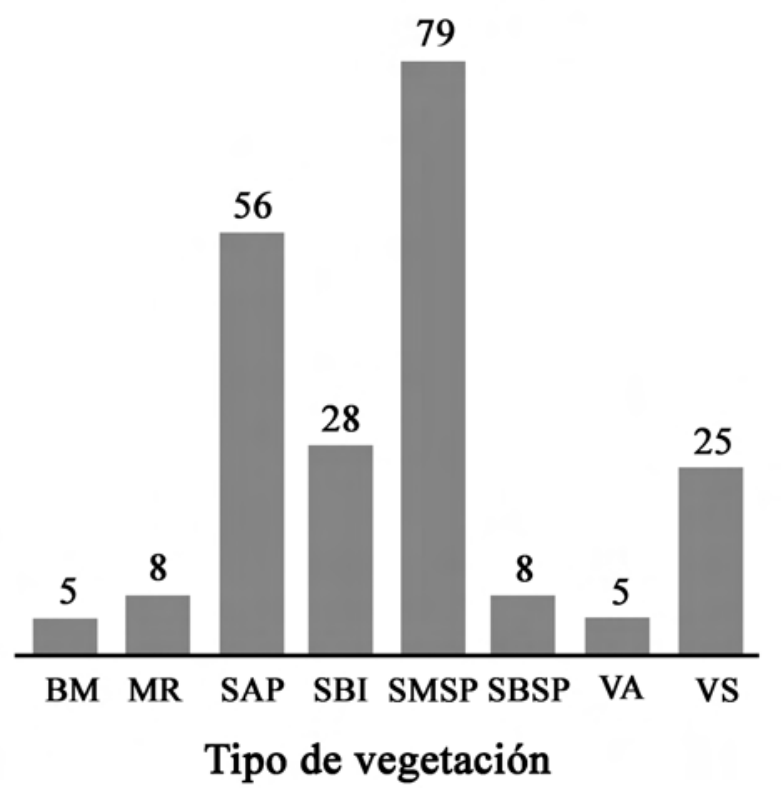

Figura 4. Número de taxa por tipo de vegetación en Tabasco. Abreviaciones de los tipos de vegetación: Bosque mesófilo (BM), manglar (MR), selva alta perennifolia (SAP), selva baja inundable (SBI), selva mediana subperennifolia (SMSP), selva baja subperennifolia (SBSP), vegetación acuática (VA) y vegetación secundaria (VS). 


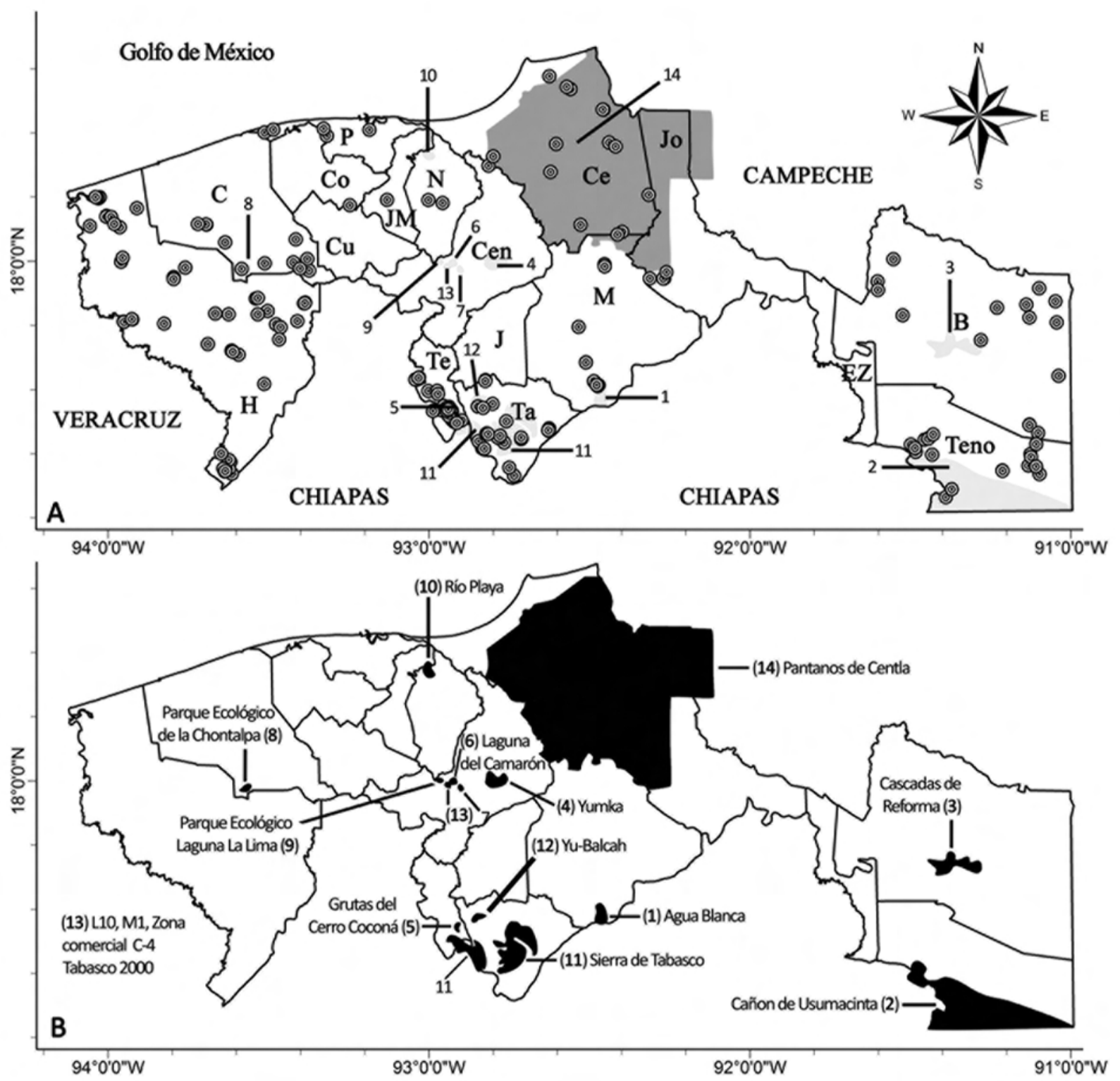

Figura. 5. Orquídeas de Tabasco. A) Distribución de Orchidaceae en los municipios y las Áreas Naturales Protegidas estatales y municipales en Tabasco. B) Localización de las Áreas Naturales Protegidas estatales y municipales. Abreviaciones de los municipios: Balancán (B), Cárdenas (C), Centla (Ce), Centro (Cen), Comalcalco (Co), Cunduacán (Cu), Emiliano Zapata (EZ), Huimanguillo (H), Jalapa (J), Jalpa de Méndez (JM), Jonuta (Jo), Macuspana (M), Nacajuca (N), Paraíso (P), Tacotalpa (Ta), Teapa (Te) y Tenosique (Teno).

frecuente Bletia purpurea, de hábito terrestre, creciendo en taludes a orilla de carretera. Entre las epifitas frecuentes están: Campylocentrum micranthum, por lo general creciendo sobre árboles de mango (Mangifera indica); Catasetum integerrimum en cercas vivas de Gliricidia sepium, en potreros y orilla de caminos; Epidendrum cardiophorum y Oncidium sphacelatum, creciendo en árboles a orilla de carretera en zonas muy abiertas. La menor diversidad de especies se registró en la selva baja subperennifolia y el manglar, con ocho especies cada una, seguido de la vegetación acuática con siete especies y el bosque mesófilo con cinco especies (Figura 4), la baja diversidad de especies reconocida para estos dos últi- mos tipos de vegetación quizás sea el resultado de un efecto de muestreo, ya que se esperaría encontrar una alta diversidad de epífitas, particularmente en el bosque mesófilo.

Especies en riesgo y registradas en Áreas Naturales Protegidas. Se reconocieron seis especies en alguna categoría de riesgo de conservación de acuerdo a la NOM-059-ECOL2010 (SEMARNAT, 2010), tres como amenazadas (Cycnoches ventricosum, Epidendrum cnemidophorum y Specklinia digitale) y tres en protección especial (Epidendrum isthmi, Stelis cobanensis y Vanilla planifolia). El 48\% (53) de las especies están presentes en seis ANP, 39 en la Sie- 
rra de Tabasco, 11 en Agua Blanca, diez en los Pantanos de Centla, seis en el Cañón de Usumacinta, una en las Cascadas de Reforma y una en el Parque Ecológico de la Chontalpa (Apéndice 1, Figura 5); en las otras ocho ANP restantes no se encontraron registros; sin embargo, no se descarta la presencia de orquídeas. De las especies consideradas en alguna categoría de riesgo, únicamente $E$. isthmi se encontró registrada dentro de una ANP (Sierra de Tabasco).

\section{Discusión}

Nuevos registros de especies. El número de especies registrado y actualizado en esta recopilación es mayor a los obtenidos en otros trabajos para la orquideoflora de Tabasco (Cuadro 1), con excepción de Cruz-Hernández (2000), en el que se indicó un mayor número de taxa (145); sin embargo, muchas de ellas corresponden a sinónimos o identificaciones incorrectas (Cuadro 1, Apéndice 1). Todos los estudios locales consultados y enfocados exclusivamente a las orquídeas de Tabasco requirieron de actualizaciones nomenclaturales y de re-determinaciones (Cuadro 1); por ejemplo, en Cowan (1983) de 46 especies registradas, 21 demandaron ser identificadas nuevamente, 11 fueron actualizadas y solamente diez se mantuvieron de acuerdo al conocimiento actual. Diversos trabajos posteriores al de Cowan (1983) se basaron en sus identificaciones y colecciones, y esto se evidencia en los nombres aplicados a las especies y los mismos ejemplares citados (p. ej., Alderete y Cappello, 1988; Alderete, 1992; Ramón-Jiménez, 1992; López-Hernández, 1994; Cruz-Hernández, 2000 y Pérez et al., 2005). Los trabajos florísticos constituyen fuentes de información de suma importancia, pues entre otros aspectos determinan el número de especies presentes en una región y permiten su correcta identificación (Atwood, 1987; Pupulin 1998; Bogarín y Pupulin, 2007). La existencia de esta información sirve de base para la realización de otro tipo de investigaciones en diversas áreas del conocimiento.

Entre los 12 nuevos hallazgos para el estado se encuentra la especie naturalizada Oeceoclades maculata, a pesar de su amplia distribución y frecuencia en diversos tipos de vegetación en el país y el Neotrópico; de igual forma sucede con Prescottia stachyodes, de hábito terrestre y también de distribución neotropical. Quizás estas especies han pasado desapercibidas en otros inventarios por su hábito terrestre y por su floración poco llamativa, que difícilmente puede observarse entre los arbustos del sotobosque. De igual forma constituye un nuevo registro Maxillariella variabilis, especie de amplia distribución y rangos altitudinales en México. Myrmecophila christinae var. christinae había sido previamente identificada por Alderete y Cappello (1988) como Myrmecophyla sp., con base en una fotografía; sin embargo, la presencia de esta especie fue confirmada con material de herbario. En cuanto a Stanhopea oculata, es una especie poco frecuente, que crece a elevaciones entre los 600 a 1,800 m, registrada en este trabajo en el municipio de Huimanguillo; Soto (2002a) la inventarió para Chiapas y la consideró como amenazada debido a la sobre recolección. Más de la mitad de los nuevos registros aquí presentados se basan en especímenes procesados de las colecciones de herbarios regionales, esto en concordancia con lo señalado por Bebber et al. (2010), que solo un pequeño número de nuevas especies se reconocen en el momento de ser recolectadas, de lo contrario, estas especies se describen posteriormente a partir de especímenes de herbario, a menudo después de un considerable lapso de tiempo después de su recolección; con lo que se resalta una vez más que los herbarios pueden ser reservorios de diversidad no descrita de floras, a pesar de que estas hayan sido ampliamente colectadas.

Distribución geográfica y áreas vecinas. En general, la orquideoflora tabasqueña es diversa en elementos de amplia distribución en el Neotrópico (Centroamérica y norte de Sudamérica), y el sureste mexicano. Tabasco, como parte de la provincia biogeográfica Golfo de México (Morrone, 2001) comparte elementos florísticos con Chiapas y Veracruz, y además con la Provincia biótica de la Península de Yucatán (Morrone, 2001). En este sentido, de los 110 taxa de orquídeas presentes en Tabasco, 100 se distribuyen también en Chiapas (Beutelspacher, 2008), 87 en Veracruz y 71 en la Península de Yucatán (Carnevali et al., 2010); asimismo, estas tres áreas y Tabasco comparten la presencia de 54 especies. No existe, al momento, registro de especies de orquídeas restringida geográficamente al estado; sin embargo, destaca la distribución de Epidendrum pachyrachis, presente en México sólo en Tabasco (Teapa) y en Oaxaca (Santa María Chimalapa; Hágsater, 2008).

Durán et al. (1988) consideraron el área política de Balancán (Tabasco) como parte de la Provincia biótica de la Península de Yucatán, al igual que el área del pie de montaña de la Sierra Norte de Chiapas. La Sierra de Tabasco forma parte de las Montañas del Norte de Chiapas, mismas, que en Tabasco tienen una mayor proporción en los municipios de Tacotalpa y Teapa. El relieve está compuesto de una topografía muy accidentada, en donde las pendientes son muy pronunciadas $(70 \%)$, lo que define a sus características como únicas en todo el estado (López-Hernández, 1994); además, las condiciones climáticas (alta humedad y precipitación) han favorecido la existencia de una alta riqueza florística. Por otra parte, la sierra de Tabasco forma parte de un amplio corredor biogeográfico que funcionó como un refugio de especies de ambientes cálidos húmedos durante el Pleistoceno (Wendt, 1989), lo que explica las afinidades florísticas de esta zona del sur de Tabasco con otras zonas de México y Centroamérica (López-Hernández, 1994).

Riqueza por tipos de vegetación. Los últimos fragmentos de selva mediana subperennifolia se localizan en la zona 
serrana de los municipios de Huimanguillo, Macuspana, Tacotalpa, Teapa y Tenosique (Castillo y Zavala, 1996). No es coincidencia que esta región de la Sierra sea la más diversa en cuanto a número de especies, esto debido a que en los fragmentos de vegetación existe una alta biodiversidad, en la que se incluyen alrededor de 2,589 especies de plantas (Pérez et al., 2005), de estas, las orquídeas presentes en los municipios de Teapa y Tacotalpa representan cerca del 50\% del total de la orquideoflora que crece en el estado. La alta diversidad especifica en esta región puede estar además relacionada con la existencia del Área Natural Protegida Parque Estatal de la Sierra (ubicado entre Teapa y Tacotalpa), en la que se registraron, en el presente estudio, 39 especies de orquídeas. Sin embargo, a pesar de la existencia de esta ANP, la pérdida de selvas altas y medianas perennifolias se ha incrementado y, en menos de 30 años, estos ecosistemas han disminuido en un 60\% (Salazar, 2000). Por otra parte, el tipo de vegetación más restringido en área natural en Tabasco probablemente sea el bosque mesófilo de montaña, el cual presenta fragmentos en las dos prominencias orográficas más importantes del estado (Castillo et al., 1995): en el Cerro de las Flores, en Huimanguillo, a $800 \mathrm{~m}$ de altitud; y muy probablemente también en el Cerro Madrigal, el cual alcanza 900 m de altitud, en Teapa (Pérez et al., 2005). El bosque mesófilo de montaña destaca por poseer una alta diversidad de especies arbóreas, epífitas, pteridofitas y trepadoras (Ortega y Castillo, 1996). Por lo que, el escaso registro de orquídeas en este tipo de vegetación resulta contradictorio a lo esperado, y conduce a pensar en la posibilidad de un efecto de recolecta insuficiente en la zona, o quizás a la poca disponibilidad de material previamente recolectado no procesado en los herbarios. De acuerdo a Zavala et al. (2003), se ha observado un incremento de la vegetación secundaria, debido al deterioro de las selvas y al abandono de tierras de cultivos y pastizales en el estado. La vegetación secundaria muestreada en Tacoltapa, en este estudio, corresponde a acahuales viejos (mayor de diez años) en los que se registró un mayor número de orquídeas ( 25 spp.) si se compara con tipos de vegetación como la selva baja perennifolia (8 spp.). Este mayor número de especies puede deberse a que muchos de estos acahuales han derivado de vegetación primaria que no sufrió un grado de alteración tan alto, y además, parte de estos acahuales se sitúan en las zonas altas de la sierras a la par de los fragmentos de selva más conservados (LópezHernández, 1994). No obstante, en la vegetación secundaria solo está presente aproximadamente el $50 \%$ del total de las especies registradas para estos fragmentos de selvas altas y medianas subperennifolias, lo que conlleva al planteamiento urgente de seguir protegiendo estos acahuales para que logren alcanzar un mayor nivel de restauración.

Estado de conservación. La Sierra Madrigal es uno de los últimos relictos con selva alta perennifolia y selva mediana subperennifolia en Tabasco y está protegida por decreto como reserva estatal (Salazar et al., 2004). Sin embargo, la degradación de estas selvas ha seguido la misma tendencia de deterioro observado para el resto del estado (Zavala y Castillo, 2003). El 48\% de las especies de orquídeas que están registradas para el estado están presentes en ANP estatales, lo que representa un dato esperanzador para promover iniciativas de conservación, reforestación y restauración de las selvas en Tabasco, y de esta forma resguardar su biodiversidad. Por otra parte, es necesario mencionar que mientras se han realizado esfuerzos por aumentar el conocimiento en general de la flora del estado, simultáneamente, ocurren procesos de deforestación, de expansión ganadera extensiva, intensificación agrícola, urbanización y explotación petrolera, que han modificado la mayoría de los ecosistemas (Sánchez y Barra, 2005). La expansión ganadera es la actividad que afectó, y continúa provocando, la reducción del 95\% del millón de hectáreas originales de selva. Ante esta situación se hace más imperioso el conocimiento de la flora y su distribución para su resguardo y conservación.

\section{Consideraciones finales}

Esta revisión permitió integrar y actualizar la información dispersa de la orquideoflora tabasqueña; sin embargo, a pesar de los avances en el conocimiento de la flora del estado y particularmente de su orquideoflora, todavía se requiere de mayores exploraciones botánicas, principalmente en los municipios de Jalpa de Méndez, Jonuta y Emiliano Zapata, que permitan tener un mayor conocimiento de la diversidad. La orquideoflora de Tabasco puede incrementarse a 134 taxa o más, considerando que el número de especies esperadas sean respaldadas con material de herbario.

\section{Agradecimientos}

A Neptalí Ramírez Marcial y Mario González Espinosa (ECOSUR) por todas las facilidades otorgadas para el desarrollo y culminación del presente trabajo de investigación. Trabajo de campo y herbario financiado por el proyecto FORDECYT 143303 "Gestión y estrategias de manejo sustentable para el desarrollo regional en la cuenca hidrográfica transfronteriza Grijalva" y por la Secretaría de Recursos Naturales y protección Ambiental del estado de Tabasco (SERNAPAM). La primera autora agradece al ECOSUR por la beca otorgada para estudios posdoctorales. El segundo autor agradece al CONACyT por la beca doctoral y posdoctoral. A las curadoras Eustolia García López (CSAT) y María de los Ángeles Guadarrama (UJAT) por las facilidades brindadas para la revisión de los especímenes. A Sergio Avendaño Reyes (XAL), Germán Carnevali y Silvia Hernández (CICY) por proporcionarnos información de los especímenes de herbario e imágenes de orquídeas de Tabasco. A Miguel Martínez-Icó (ECOSUR) por su colaboración durante la revisión del material en los herbarios y en las 
salidas de campo. Al equipo de viveros del ECOSUR-SCL, Alfonso Luna, Henri Castañeda, Soledad Gómez y Zenaida Hernández por su colaboración durante las salidas de campo a Tabasco. A Jesús Chi residente del proyecto FORDECYT 143303 en Tabasco, por su colaboración para la realización de las salidas de campo en las comunidades. A José Luis Tapia Muñoz (CICY) por sus comentarios y sugerencias al manuscrito. A Gustavo Romero González (AMES) por sus comentarios y sugerencias al resumen. A Germán Carnevali por la identificación de las especies de Myrmecophila y por facilitarnos la fotografía de Chysis limminghei. Lizandro Peraza por identificar las especies de Polystachya. Ricardo Balam por compartir sus registros de Lophiaris lindenii. Gustavo Romero-González (AMES) proporcionó información de Aspydogyne querceticola. Hermilo Cruz García y Nancy Zamora (ECOSUR) por la literatura suministrada. A las personas de las comunidades de los ejidos vecinos a Tapijulapa que nos guiaron y acompañaron en las salidas de campo. Agradecemos a los dos revisores anónimos por sus valiosos comentarios al manuscrito.

\section{Literatura citada}

Alderete C.A. 1992. Listado complementario de las Orquídeas de Tabasco. Revista Agrociencia del Colegio de Postgraduados, Serie Recursos Naturales Renovables 2:106-123.

Alderete C.A. y Cappello G.S. 1988. Orquídeas de Tabasco. Instituto Nacional de Insvestigaciones sobre Recursos Bióticos, Instituto de Cultura de Tabasco, Villahermosa.

Atwood J.T. 1987. The vascular flora of la Selva Biological Station, Costa Rica: Orchidaceae. Selbyana 10:76-145.

Balam R., Carnevali G., Cetzal-Ix W. y Duno R. 2011. Lophiaris tapiae, a new species in the Lophiaris oerstedii complex (Orchidaceae) from the Yucatan Peninsula, Mexico. Acta Botanica Mexicana 97:17-29.

Beutelspacher B.C.R. 2008. Catálogo de las orquídeas de Chiapas. Lacandonia 2:25-122.

Bezaury-Creel J.E., Torres J.F. y Moreno N. 2007. Base de datos geográfica de áreas naturales protegidas estatales, del Distrito Federal y municipales de México para el análisis de vacíos y omisiones en conservación. The Nature Conservancy, Pronatura, Comisión Nacional para el Conocimiento y Uso de la Biodiversidad, Comisión Nacional de Áreas Naturales Protegidas, México, D.F.

Bebber D.P., Carine M.A., Wood J.R.I., Wortley A.H., Harris D.J., Prance G.T., Davidse G., Paige J., Pennington T.D., Robson N.K.B. y Scotland R.W. 2010. Herbaria are a major frontier for species discovery. Proceedings of the National Academy of Sciences of United States of America 107:22169-22171

Bogarín D. y Pupulin F. 2007. Las orquídeas del Parque Nacional Barra Honda, Guanacaste, Costa Rica. Lankesteriana 7:446449.

Carnevali F.C.G., Tapia-Muñoz J.L., Duno de Stefano R. y Ramírez M.I. 2010. Flora Ilustrada de la Península de Yucatán: Listado Florístico. Centro de Investigación Científica de Yucatán, A.C., Mérida.

Carnevali G., Cetzal-Ix W., Balam R., Leopardi C. y Romero-
González G.A. 2013. A combined evidence phylogenetic recircumscription and a taxonomic revision of Lophiarella (Orchidaceae: Oncidiinae). Systematic Botany 38:46-63.

Carnevali F.C.G., Tapia-Muñoz J.L., Jiménez-Machorro R., Sánchez-Saldaña L., Ibarra-González L., Ramírez I.M. y Gómez M.P. 2001. Notes on the flora of the Yucatan Peninsula II: A synopsis of the orchid flora of the Mexican Yucatan Peninsula and a tentative checklist of the Orchidaceae of the Yucatan Peninsula Biotic Province. Harvard Papers in Botany 5:383-466.

Castillo O., Olan D., Narváez U., Calzada A., Mendieta M. y Ramos R. 1995. El Bosque mesofilo de montaña en el municipio Huimanguillo, Tabasco. XIII Congreso Mexicano de Botánica: Diversidad Vegetal de México. Cuernavaca, Morelos. Libro de Resúmenes. Universidad Autónoma del Estado de Morelos, Sociedad Botánica de México, México, D.F.

Castillo G.O. y Zavala C.J. 1996. Fisiografía, recursos vegetales y alternativas de manejo en el Parque Estatal Agua Blanca, Tabasco. Universidad y Ciencia 12:63-70.

Cervantes L., Hágsater E. y Soto M. 2002. Epidendrum nocturnum Jacq. Icones Orchidacearum fascicles 5-6, Orchids of Mexico Parts 2-3, pl. 578, Herbario AMO, México, D.F.

Cetzal-Ix W. y Balam N.R. 2012. The identity and distribution of Lophiaris carthagenensis (Orchidaceae, Oncidiinae): two centuries of confusion. Journal of the Torrey Botanical Society 139:9-25.

Cetzal-Ix W. y Carnevali F.C.G. 2010. A revision of Cohniella Pfitzer (Orchidaceae) in Mexico. Journal of the Torrey Botanical Society 137:180-213.

Chase M.W. 2009. Subtribe Oncidiinae. En: Pridgeon A.M., Cribb P.J., Chase M.W. y Rasmussen F.N. Eds. Genera Orchidacearum. Epidendroideae. Volumen 5. Epidendroideae (Part two), pp. 211-394, Oxford University Press, Oxford.

Cowan C.P. 1983. Listados Florísticos de México. I. Flora de Tabasco. Instituto de Biología, Universidad Nacional Autónoma de México, México, D.F.

Cruz-Hernández J.A. 2000. Las orquídeas de Tabasco y sus afinidades ecológicas. Tesis de licenciatura, Centro Regional Universitario del Sureste, Universidad Autónoma Chapingo, Tabasco. 87 pp.

Díaz-Jiménez P. 2007. Flora epífita de los manglares de Tabasco, México. Kuxulkab’ 25:11-17.

Durán R., Trejo-Torres J.C. y Ibarra-Manríquez G. 1998. Endemic phytotaxa of the Yucatán Peninsula. Harvard Papers of Botany 3:263-314.

Espejo S.A. y López-Ferrari A.R. 1997. Las Monocotiledóneas Mexicanas, una Sinopsis Florística. 1. Lista de Referencia. Parte VII. Orchidaceae 1. Consejo Nacional de la Flora de México, A.C., Universidad Autónoma Metropolitana Iztapalapa, Comisión Nacional para el Conocimiento y Uso de la Biodiversidad. México, D.F.

Espejo S.A. y López-Ferrari A.R. 1998. Las Monocotiledóneas Mexicanas, una Sinopsis Florística. 1. Lista de Referencia. Parte VIII. Orchidaceae 2. Consejo Nacional de la Flora de México, A.C., Universidad Autónoma Metropolitana Iztapalapa, Comisión Nacional para el Conocimiento y Uso de la Biodiversidad. México, D.F.

Hágsater E. 2008. Epidendrum pachyrachis Ames. Icones Orchidacearum fascicle 11, The Genus Epidendrum Part 7, pl. 1152, Herbario AMO, México, D.F.

Hágsater E. y Soto M. 2002. Ponera striata Lindl. Icones Orchi- 
dacearum facicles 5-6, Orchids of Mexico Part 2-3, pl. 646, Herbario AMO, México, D.F.

Halbinger F. y Soto M. 1997. Laelias of Mexico. Orquídea (Méx.) 15:1-160.

Hanan-Alipi A.M. 1997. Análisis florístico de la Sierra El Madrigal, Teapa, Tabasco. Tesis de licenciatura, Facultad de Ciencias, Universidad Nacional Autónoma de México, México, D.F. 50 pp.

Holmgren P.K., Holmgren N.H. y Barnett L.C. 1990. Index Herbariorum. Part I: Herbaria of the World (Regnum Vegetabile, vol. 120). New York Botanical Garden Press, Nueva York.

INEGI. Instituto Nacional de Estadística y Geografía. 2007. Anuario Estadístico del estado de Tabasco, Edición 2007. <http:// www.inegi.org.mx/est/contenidos/espanol/sistemas/Aee07/ estatal/tab/index.htm> (consultado 15 junio 2013).

INEGI. Instituto Nacional de Estadística y Geografía. 2011. Vegetación de Tabasco. <http://mapserver.inegi.gob.mx/geografia /espanol/estados/tab/agri.cfm?c=444\&e=07> (consultado 22 enero 2013).

Jiménez M.R. y Hágsater E. 2010. Epidendrum cardiophorum Schltr. Icones Orchidacearum fascicle 13, The Genus Epidendrum Part 9, pl. 1311, Herbario AMO, México, D.F.

Judd W.S., Campbell C.S., Kellogg E.A., Stevens P.F. y Donoghue M.J. 2008. Plant Systematics: A Phylogenetic Approach, $3^{\text {rd }}$ ed. Sinauer Associates, Sunderland.

López-Hernández E.S. 1994. La vegetación y la flora de la Sierra de Tabasco (municipios de Tacotalpa y Teapa) México. Tesis licenciatura, División Académica de Ciencias Biológicas, Universidad Juárez Autónoma de Tabasco, México, Villahermosa. $88 \mathrm{pp}$.

Morrone J.J. 2001. Biogeografía de América Latina y el Caribe. Manuales y Tesis SEA 3, Zaragoza.

Noguera-Savelli E. 2010. Revisión del género Brassavola R. Br. (Orchidaceae) para Venezuela. Ernstia 20:169-192.

Noguera-Savelli E. y Cetzal-Ix W. 2013. Primer registro de Cycnoches ventricosum (Orchidaceae: Catasetinae) para Tabasco, México. Botanical Sciences 91:233-236.

Novelo A. y Ramos L. 2005. Vegetación acuática. En: Bueno J., Álvarez F. y Santiago S. Eds. Biodiversidad del Estado de Tabasco, pp. 111-144, Instituto de Biología, Universidad Nacional Autonoma de México, Comisión Nacional para Uso y Conocimiento de la Biodiversidad, México, D.F.

Ormerod P. 2013. Studies of Neotropical Goodyerinae (Orchidaceae) 5. Harvard Papers in Botany 18:51-60.

Ortega E.F. y Castillo C.G. 1996. El bosque mesófilo de montaña y su importancia forestal. Ciencias 43:32-39.

Peraza-Flores L.N., Carnevali F.C.G. y Romero-González G.A. 2011. Taxonomic notes in American Polystachya (Orchidaceae): the identity of $P$. foliosa (Hook.) Rchb.f. and the reestablishment of P. caracasana Rchb.f. Journal of the Torrey Botanical Society 138:366-380.

Pérez L.A., Sousa S.M., Hanan A.M., Chiang F. y Tenorio P. 2005. Vegetación Terrestre. En: Bueno J., Álvarez E. y Santiago S. Eds. Biodiversidad del Estado de Tabasco, pp. 65-110, Instituto de Biología, Universidad Nacional Autonoma de México, Comisión Nacional para Uso y Conocimiento de la Biodiversidad, México, México, D.F.

Pupulin F. 1998. Orchid florula of Parque Nacional Manuel Antonio Quepos, Costa Rica. Revista de Biología Tropical 46:96110317.

Ramón-Jiménez V. 1992. Flora de angiospermas epífitas en la ve- getación riparia del río Puyacatengo, Teapa Tabasco, México. Tesis licenciatura, División Académica de Ciencias Biológicas, Universidad Juárez Autónoma de Tabasco, Villahermosa. $52 \mathrm{pp}$.

Salazar C.E.C. 2000. Dinámica de Uso del Suelo en el Parque Estatal de la Sierra, Tabasco (1972-1995). Universidad Juárez Autónoma de Tabasco, Villahermosa.

Salazar G.A. 2002. Gongora leucochila Lem. Icones Orchidacearum fascicles 5-6, Orchids of Mexico Parts 2-3, pl. 587, Herbario AMO, México, D.F.

Salazar G.A. 2013. Two additions to the Mexican Orchid flora. Revista Mexicana de Biodiversidad 84:378-380.

Salazar C.E.C., Zavala C.J., Castillo A.O. y Cámara A.R. 2004. Evaluación espacial y temporal de la vegetación Sierra Madrigal, Tabasco, México (1973-2003). Investigaciones Geográficas, Boletín del Instituto de Geografía, UNAM 54:7-23.

Sánchez A.J. y Barba E. 2005. Biodiversidad de Tabasco. En: Bueno J., Álvarez E. y Santiago S. Eds. Biodiversidad del Estado de Tabasco, pp. 1-16, Instituto de Biología, Universidad Nacional Autonoma de México, Comisión Nacional para Uso y Conocimiento de la Biodiversidad, México, D.F.

Sánchez L. 2002. Epidendrum chlorocorymbos Schltr. Icones Orchidacearum fascicles 5-6, Orchids of Mexico Parts 2-3, pl. 570, Herbario AMO, México, D.F.

Sánchez S.L. y Hágsater E. 2010. Epidendrum ciliare L. Icones Orchidacearum fascicle 13, The Genus Epidendrum Part 9, pl. 1317, Herbario AMO, México D.F.

Sánchez S.L. y Hágsater E. 2010. Epidendrum galeottianum A.Rich \& Galeotti. Icones Orchidacearum fascicle 13, The Genus Epidendrum Part 9, pl. 1335, Herbario AMO, México D.F.

Sánchez S.L. y Hágsater E. 2010. Epidendrum martinezii L.Sánchez \& Carnevali. Icones Orchidacearum fascicle 13, The Genus Epidendrum Part 9, pl. 1355, Herbario AMO, México D.F.

Santiago E. y Hágsater E. 2010. Epidendrum cnemidophorum Lindl. Icones Orchidacearum fascicle 13, The Genus Epidendrum Part 9, pl. 1322, Herbario AMO, México D.F.

SEMARNAT. Secretaría del Medio Ambiente y Recursos Naturales. 2010. Norma Oficial Mexicana NOM-059-SEMARNAT2010 Protección ambiental-Especies nativas de México de flora y fauna silvestres-Categorías de riesgo y especificaciones para su inclusión, exclusión o cambio-Lista de especies en riesgo.

Solano R. 1993. El género Stelis Sw. (Orchidaceae: Pleurothallidinae) en México. Orquídea (Méx.) 13:1-112.

Solano R. 2002. Acianthera hondurensis (Ames) Pridgeon \& M.W.Chase. Icones Orchidacearum fascicles 5-6, Orchids of Mexico Parts 2-3, pl. 508, Herbario AMO, México, D.F.

Solano R. 2008. Specklinia digitale (Luer) Pridgeon \& M.W.Chase. Icones Orchidacearum fascicle 10, Orchids of Mexico Part 4, pl. 1091, Herbario AMO, México D.F.

Soto A.M.A. 1988. Listado actualizado de las orquídeas de México. Orquídea (Méx.) 11:233-277.

Soto M. 2002a. Stanhopea oculata (G.Lodd.) Lindl. Icones Orchidacearum fascicles 5-6, Orchids of Mexico Parts 2-3, pl. 677, Herbario AMO, México, D.F.

Soto M. 2002b. Vanilla insignis Ames. Icones Orchidacearum fascicles 5-6, Orchids of Mexico Parts 2-3, pl. 700, Herbario AMO, México, D.F.

Soto M. 2008. Chysis bractescens Lindl. Icones Orchidacearum fascicle 10, Orchids of Mexico Part 4, pl. 1012, Herbario AMO, México D.F. 
Soto M. 2008. Prosthechea radiata (Lindl.) W.E.Higgins. Icones Orchidacearum fascicle 10, Orchids of Mexico Part 4, pl. 1072, Herbario AMO, México D.F.

Soto A.M.A. y Dressler R.L. 2010. A revision of the Mexican and Central American species of Vanilla Plumier ex Miller with a characterization of their ITS region of the nuclear ribosomal DNA. Lankesteriana 9:285-354.

Soto A.M.A., Solano G.R. y Hágsater E. 2007. Risk of extintion and patterns of diversity loss in Mexican orchids. Lankesteriana 7:114-121.

Velázquez-Villegas G. 1994. Los recursos hidráulicos del Estado de Tabasco. Universidad Juárez Autónoma de Tabasco, Centro de Investigación de la Difusión Académica de Ingeniería y Tecnología, Villahermosa.

Recibido:19 de agosto de 2013

Aceptado: 2 de noviembre de 2013
Wendt T. 1989. Las selvas de Uxpanapa, Veracruz-Oaxaca, México. Evidencia de refugios florísticos cenozoicos. Anales del Instituto de Biología Universidad Nacional Autónoma de México, Serie Botánica 58:29-54.

Zavala C.J. y Castillo A.O. 2003. Uso del suelo y manejo en los cordones litorales de la Reserva de la Biosfera Pantanos de Centla, Tabasco. Universidad y Ciencia 19:17-33.

Zavala C.J., Ramos R.R., Castillo A.O. y Ortíz C.A.I. 2003. Cambio de uso del suelo. En: Zavala C.J., Gutiérrez C.M.C. y Palma-López D.J. Eds. Impacto Ambiental en las Tierras del Campo Petrolero Samaría, Tabasco, pp. 33-52, Colegio de Postgraduados, Campus Tabasco, Consejo de Ciencia y Tecnología del Estado de Tabasco, Consejo Nacional de Ciencia y Tecnología, Villahermosa. 
Apéndice 1. Lista de las orquídeas de Tabasco, México. Municipios: Balancán (B), Cárdenas (C), Centla (Ce), Centro (Cen), Comalcalco (Co), Cunduacan (Cu), Emiliano Zapata (EZ), Huimanguillo (H), Jalapa (J), Jalpa de Méndez (JM), Jonuta (Jo), Macuspana (M), Nacajuca (N), Paraíso (P), Tacotalpa (Ta), Teapa (Te) y Tenosique (Teno). Tipo de vegetación: Bosque mesófilo (BM), Manglar (MR), Selva alta perennifolia (SAP), Selva mediana subperennifolia (SMSP), Selva baja subperennifolia (SBSP), Selva baja inundable (SBI), Vegetación acuática (VA) y Vegetación secundaria (VS). Hábito: Epífita, Terrestre, Rupícola. Referencias: Cowan (1983) (1), Alderete y Cappello (1988) (2), Soto (1988) (3), Alderete (1992) (4), Ramón-Jiménez (1992) (5), López-Hernández (1994) (6), Hanan-Alipi (1997) (7), Espejo y López-Ferrari (1997, 1998) (8), Cruz-Hernández (2000) (9), Carnevali et al. (2001) (10), Pérez et al. (2005) (11), Novelo y Ramos (2005) (12), Carnevali et al. (2010) (13). El asterisco $\left(^{*}\right)$ en las abreviaciones de los autores indica que no poseen un ejemplar de herbario o fotografía de respaldo. Estado de Conservación de acuerdo a la Nom059-SEMARNAT-2010. Áreas Naturales Protegidas (ANP): Agua Blanca (1), Cañón de Usumacinta (2), Cascadas de Reforma (3), Centro de interpretación de la Naturaleza (Yumka) (4), Grutas del Cerro Coconá (5), Laguna de las llusiones (6), Laguna del Camarón (7), Parque Ecológico de la Chontalpa (8), Parque Ecológico Laguna La Lima (9), Río Playa (10), Sierra de Tabasco (11), Yu-Balcah (12), L10, M1, Zona comercial C-4 Tabasco 2000 (13), Pantanos de Centla (14).

Acianthera hondurensis (Ames) Pridgeon \& M.W.Chase, Lindleyana 16(4): 244. 2001. Pleurothallis hondurensis Ames, Sched. Orch. 7: 20-21, t. 15. 1924. (Te; SAP; Epífita; Guadarrama \& Ortíz 95.5.101, UJAT). Referencias: 2, Solano (2002: 508). Comentarios: Registrado por Alderete y Cappello (1988: 63) como Pleurothallis sp.

Anathallis sertularioides (Sw.) Pridgeon \& M.W.Chase, Lindleyana 16(4): 250. 2001. Epidendrum sertularioides Sw., Prodr. 122. 1788. (H; SAP; Epífita; Magaña et al. 1738, UJAT). Referencias: 7, 11.

Anathallis yucatanensis (Ames \& C.Schweinf.) Solano \& Soto Arenas, Icon. Orchid. 5-6: 10. 2003. Pleurothallis yucatanensis Ames \& C.Schweinf., Bot. Mus. Leafl. 1(2): 4. 1932. Specklinia yucatanensis (Ames \& C.Schweinf.) Pridgeon \& M.W.Chase, Lindleyana 16(4): 260. 2001. (B; SMSP; Epífita; Valdivia 2081, XAL). Comentarios: Nuevo registro.

Aspydogyne querceticola (Lindl.) Meneguzzo, Orquidario 26(3): 90. 2012. Platythelys maculata (Hook.) Garay, Bradea 2(28): 197. 1977. Physurus maculatus Hook., Bot. Mag. 88: t. 5305. 1862. Platythelys vaginata (W.J.Hook) Meneguzzo, Orquidario 26(3): 91. 2012. Sinonimia completa ver Ormerod (2013). (M, Ta, Te; SAP, SMSP; Terrestre; Noguera \& Savelli 957, CH; Castillo 760, Hanan 342, Guadarrama et al. 1063, Hernández 355, Magaña 1483, UJAT). Referencias: 3, 7, 8, 9, 10, 11, 13. ANP: 11. Comentarios: Citado por Soto (1988), Espejo y López-Ferrari (1997, 1998), Carnevali et al. (2001, 2010) y Pérez et al. (2005) como Platythelys vaginata; por Hanan-Alipi (1997) como P. maculata; y por Cruz-Hernández (2000) como P. vaginata y P. maculata.

Beloglottis costaricensis (Rchb.f.) Schltr., Beih. Bot. Centralbl., Abt. 37(2/3): 365. 1920. Spiranthes costaricensis Rchb.f., Bonplandia 3(15/16): 214. 1855. (Ta, M; SMSP, SBSP; Terrestre; Noguera \& Cetzal 690, CH; Ortíz et al. 7101, Zamudio \& Guadarrama 850, UJAT). Referencias: 9, 11*. ANP: 1.

Bletia purpurea (Lam.) DC., Mém. Soc. Phys. Genève 9(1): 97-98. Limodorum purpureum Lam., Encycl. 3(2): 515. 1791. (B, Ce, Cen, Co, H, Jo, M, N, Ta, Te, Teno; VA, VS; Terrestre; Cowan 2524, Magaña 8, 30, Magaña \& Cowan 2878, CSAT; Fernández \& Zamudio 1008, CSAT, UJAT, XAL; Menéndez et al. 438, MO, XAL; Contreras 38, Cruz 36, García \& Ramírez 75, Guadarrama 1012, Guadarrama et al. 5048, Guadarrama \& Magaña 1091, Guadarrama \& Ortíz 3993, 95.5 .62 (sic), Magaña \& Guadarrama 2220, Novelo \& Ramos 2206, 2247, 2302, 2584, 3532, Ortíz 5131, Ramírez s.n., Sol 989, UJAT; Guadarrama et al. 6399, UJAT, XAL). Referencias: 1, 2, 3*, 4, 8*, 9, 10*, 11, 12, 13*. ANP: 2, 14. Comentarios: Cowan (1983) identificó un espécimen como B. palmeri S.Watson que corresponde a B. purpurea y que posteriormente fue citado por Alderete (1992) y Cruz-Hernández (2000).

Brassavola appendiculata A.Rich. \& Galeotti, Ann. Sci. Nat., Bot., sér. 3. 3: 23. 1845. (B, Te; SAP, SMSP; Epífita; Cruz \& Ortíz 33, UJAT; Ortega \& Ortíz 2507, XAL). Referencias: 4* 6, 9. Comentarios: Estudios previos habían registrado esta especie como B. cucullata (L.) R.Br. Recientemente, Noguera-Savelli (2010) aclaró la identidad de esta especie y reconoció las poblaciones presentes desde México hasta Nicaragua bajo este nombre.

Brassavola nodosa (L.) Lindl., Gen. Sp. Orchid. Pl. 114-115. 1831. Epidendrum nodosum L., Sp. Pl. 2: 953. 1753. (C, Co, P; MR; Epífita; Domínguez 6, Moreno \& Castillo 14, CSAT; Díaz 218, 221, Magaña 2439, Ortíz et al. 2197, UJAT). Referencias: 2, 3*, $4,8,9,11,12$.

Brassia maculata R. Br., Hort. Kew. (ed. 2) 5: 215. 1813. (B, Te, Teno; SBI; Epífita). Referencias: 2, 4, 9, 11*. Comentarios: Registro basado en la fotografía de Alderete y Cappello (1988: 26). 
Camaridium densum (Lindl.) M.A.Blanco, Lankesteriana 7(3): 520. 2007. Maxillaria densa Lindl., Edwards's Bot. Reg. 21 : t. 1804. 1835. (Ta; SAP; Epífita; Cruz 19, UJAT). Referencia: 9.

Camaridium pulchrum Schltr., Repert. Spec. Nov. Regni Veg. 10(248-250): 251. 1911. Maxillaria pulchra (Schltr.) L. O. Williams ex Correll, Lloydia 10(4): 212. 1947. (H, Te; SAP, SMSP; Epífita; Sol et al. 1091, Zamudio 812, UJAT). Referencias: 5, 7, 9, 11. ANP: 11.

Campylocentrum micranthum (Lindl.) Rolfe, Orchid Rev. 11(128): 245. 1903. Angraecum micranthum Lindl., Edwards's Bot. Reg. 21: t. 1772. 1835. (M, Ta, Te; SAP, SMSP, VS; Epífita; Noguera \& Cetzal 922, CH; Cowan et al. 3485, CICY, CSAT; Cowan et al. 2862, CSAT; Cruz 161, Guadarrama 95.5.48, Hanan et al. 296, Ortíz et al. 7088, Ramón 176, UJAT). Referencias: 1, 2, $3^{*}, 4,5^{*}, 6,7,8^{*}, 9,11,13^{*}$. ANP: 11 .

Catasetum integerrimum Hook., Bot. Mag. 67: t. 3823. 1840. (B, Ce, Co, H, J, M, Ta, Te, Teno; MR, SAP, SMSP, VS; Epífita; Noguera \& Cetzal 925, CH; Castillo \& Ramos 298, Magaña \& Zamudio 361, CSAT; Valdivia 2095, MO, XAL; Díaz 210, Guadarrama et al. 4107, Magaña 1305, 1635, 1858, Magaña \& Ortíz 2383, Novelo \& Ramos 3127, Ramón 222, UJAT; Magaña 1305, UJAT, XAL; Alderete 137, Ventura 20379, XAL). Referencias: 1, 2, 3*, 4, 5*, 6*, 7, 8*, 9, 11, 12,13*. ANP: 11, 14. Comentarios: Registrado por Cowan (1983) como C. maculatum Kunth.

Christensonella uncata (Lindl.) Szlach., Mytnik, Górniak \& Śmiszek, Polish Bot. J. 51(1): 59. 2006. Maxillaria uncata Lindl., Edwards's Bot. Reg. 23: sub t. 1986. 1837. Maxillaria macleei Bateman ex Lindl., Edwards's Bot. Reg. 26: misc. 70.1840. Christensonella macleei (Bateman ex Lindl.) Solano, Acta Bot. Mex. 97: 51. 2011. (Ta, Te; SAP, SMSP; Epífita; Noguera \& Cetzal 1035, CH; Cruz 156, García s.n., Magaña 2123, Mendieta 5, Ruíz et al. 71, UJAT). Referencias: 2, 3*, 4, 5*, 6, 7, 8*, 10, 11, 13*. ANP: 11. Comentario: Registrado por Hanan-Alipi (1997) y Pérez et al. (2005) como M. macleei; Cruz-Hernández (2000) registró como especies distintas a M. macleii y M. uncata.

Chysis bractescens Lindl., Edwards's Bot. Reg. 26: misc. 61. 1840. (Ta, Te; SAP, SMSP; Epífita; Noguera \& Cetzal 966, CH; Guadarrama 95.5.65, Ortíz s.n., UJAT). Referencias: 1, 2, 4, 5*, 7*, 9, 11*, Soto (2008: 1012). ANP: 11. Comentarios: Registrado por Cowan (1983) como Chysis sp.

Chysis limminghei Linden \& Rchb.f., Berliner Allg. Gartenzeitung 26: 308. 1858. (Jo; SMSP; Epífita; Fernández-Nava et al. 1475, AMO, CSAT, MO). Referencias: 3*, 4, 9, 11. ANP: 11.

Coelia triptera (Sm.) G.Don ex Steud., Nomencl. Bot. (ed. 2) Editio secunda 1: 394. 1840. Epidendrum tripterum Sm., Icon. Pict. PI. Rar. t. 14. 1793. (Ta; SAP; Epífita; Hernández 402, UJAT). Referencia: (Te) 5*, 7*, 9*, 11*. Comentarios: Estudios anteriores han señalado la presencia de esta especie en Tabasco con base en Ramón-Jiménez (1992), sin embargo, no estaba respaldado por un ejemplar de herbario o fotografía. Aquí se confirma su presencia.

Cohniella ascendens (Lindl.) Christenson, Lindleyana 14(4): 177. 1999. Oncidium ascendens Lindl., Edwards's Bot. Reg. 28: sub t. 4. 1842. Trichocentrum ascendens (Lindl.) M.W.Chase \& N.H.Williams, Lindleyana 16(2): 137. 2001. (B, C, H, Ta, Te, Teno; SAP, SMSP, VS; Epífita; Noguera \& Cetzal 996, CH; 21608, Guadarrama 95.3.34, Rico et al. 762, Ventura s.n., MEXU; Calónico et al. 21480, MEXU, UJAT; García et al. 32, Guadarrama 95.3.34, Magaña 1922, Mendieta 13, Ortíz 9, 2152, Rosa 42, Zamudio 214, UJAT; Magaña \& Zamudio 139, UJAT, XAL; Calzada 2221, Valdivia 1944, 2049, 2076, Ventura 21384, XAL). Referencias: (Co, J, M) 1, 2, 3*, 4, 5*, 6, 7, 8*, 9, 11, 13*, Cetzal-Ix y Carnevali (2010). ANP: 11. Comentarios: Identificado incorrectamente por Alderete y Cappello $(1988: 60,61)$ como O. luridum Lindl. y adicional como O. ascendens, respectivamente; Alderete (1992) citó $O$. ascendens y O. cebolleta Jacq.; sin embargo, este último nombre aplicado no se puede corroborar su identificación, ya que su estudio carece de ejemplares de herbario y fotografías.

Cycnoches egertonianum Bateman, Orchid. Mexico \& Guatemala t. 40. 1842. Cycnoches glanduliferum A.Rich. \& Galeotti ex Hemsl., Gard. Chron., n.s., 11: 268. 1879. Cycnoches stelliferum Lodd., Orchideae 15. 1844. (Te; SAP; Epífita; Richard 1214, K, P). Referencias: 3*, 8, 9. Comentarios: Identificado por Alderete (1992) como C. stelliferum. Esta especie se conoce en Tabasco únicamente del holotipo de C. glanduliferum.

Cycnoches ventricosum Bateman, Orchid. Mexico \& Guatemala t. 5. 1837. (Ta; SAP, SMSP; Epífita; Noguera \& Cetzal 1039, CICY, CH). Referencia: Noguera-Savelli y Cetzal-Ix (2013). Comentarios: Amenazada. 
ORChiDACEAE DE TABASCO

Apéndice 1. Continuación

Cyrtopodium macrobulbon (La Llave \& Lex.) G.A.Romero \& Carnevali, Harvard Pap. Bot. 4(1): 331, f. 2-4. 1999. Epidendrum macrobulbon La Llave \& Lex., Nov. Veg. Descr. 42. 1825. (Teno; VS; Terrestre; Jiménez et al. 758, UJAT). ANP: 2. Comentarios: Nuevo registro.

Dichaea panamensis Lindl., Gen. Sp. Orchid. PI. 209. 1833. (Ta, Te; SMSP; Epífita; Noguera \& Cetzal 982, CH; Guadarrama \& Ortíz 95.5.66, Sol 237, UJAT). Referencias: 5*, 9, 11. ANP: 11.

Dimerandra emarginata (G.Mey.) Hoehne, Bol. Agric. (Sao Paulo) 34: 618. 1934. Oncidium emarginatum G. Mey., Bol. Agric. (Sao Paulo) 34: 618. 1934. (C, H, Ta, Te; SAP, SMSP, SBSP, VS; Epífita; Cowan 1862, CAS, CSAT; Noguera \& Savelli 1023, CH; Cruz et al. 8, Magaña \& Acosta 1928, Magaña \& Ortíz 2389, Medina-Hernández s.n., Mendieta 7, Ortíz et al. 2133, Ramón 177, UJAT). Referencia: 1, 4, 5*, 7, 9, 11, 13*. ANP: 11. Comentarios: Identificado por Cowan (1983) como Epidendrum verrucosum var. mignanthum Ames \& Correll y citado por Alderete (1992) y Cruz-Hernández (2000), estos dos últimos autores adicionalmente registraron a $D$. emarginata.

Encyclia alata (Bateman) Schltr., Orchideen 207. 1914. Epidendrum alatum Bateman, Orchideen 207. 1914. (B, C, Te; SBI, SBSP, SMSP; Epífita; Magaña \& Curiel 974, CICY, CSAT, XAL; Zamudio 55, CSAT, MEXU, UJAT; Guadarrama 6980, Ortíz et al. 2169 , Sol et al. 1094, UJAT). Referencias: (Co, P, Teno) 1, 2, 3*, 4, 5*, 6, 7, 8*, 9, 10, 11, 13*. ANP: 11. Comentarios: Identificado por Cowan (1983) como Epidendrum sp.

Encyclia bractescens (Lindl.) Hoehne, Arq. Bot. Estado São Paulo 2(6): 150. 1952. Epidendrum bractescens Lindl., Edwards's Bot. Reg. 26: misc. 58. 1840. (B, Te, Teno; SMSP, VS; Epífita; Sol 1087, MEXU, UJAT; Ramón 49, UJAT; Alderete 147, Valdivia 2073, XAL). Referencias: 1, 2, 4, 5*, 7, 9, 11. Comentarios: Identificado por Cowan (1983) como Cymbidium sp. ANP: $2,11$.

Encyclia guatemalensis (Klotzsch) Dressler \& G.E.Pollard, Phytologia 21(7): 437. 1971. Epidendrum guatemalense Klotzsch, Allg. Gartenzeitung 20(32): 250. 1852. Encyclia belizensis (Rchb.f.) Schltr., Beih. Bot. Centralbl., Abt. 2, 36(2): 471. 1918. (B, Teno; SMSP, VS; Epífita; Matuda 3111, AMES, CAS; Cruz \& Ortíz 32, UJAT; Alderete 130, XAL). Referencias: 1, 2, 3*, 4, 5*, 8*, 9, 11. Comentarios: Registrado por Cowan (1983) como E. alata; por Soto (1988) como E. belizensis ssp. belizensis (Rchb. f.) Schltr.; Cruz-Hernández (2000) lo registró bajo E. belizensis y E. guatemalensis. Los demás autores lo incluyeron como E. belizensis.

Epidendrum cardiophorum Schltr., Repert. Spec. Nov. Regni Veg. Beih. 9(208-210): 214. 1911. (B, H, Ta, Te, Teno; SAP, SBI, SMSP, VS; Epífita; Matuta 3317, AMES, K-L, MEXU; MICH, SEL; Matuda 3114, AMES, MICH; Ventura 20654, AMO; Cowan 1704, CAS, CSAT, CICY; Cowan 3260, CAS, CSAT, ENCB, MEXU; Noguera \& Cetzal 1030, CH; Sánchez 836, CORU; Santana 357, AMO; Valdivia 2093, MEXU, XAL; Valdivia 2058, MO, XAL; Cruz \& Ortíz 24, Madrigal 15, Magaña 1306, 1857, Mares 67, Pérez 12, Ramón 178, Sol 543, 956, Zamudio 212, 457, 1412, Santana 357, UJAT). Referencia: (Cen, M, P) 1, 2, 4, 5* 6, 7, 9, 13*, Jiménez y Hágsater (2010: 1311). ANP: 1, 2, 11. Comentarios: Registrado por Cowan (1983) como Epidendrum sp., E. anceps y E. rigidum; Alderete y Cappello (1988), Alderete (1992), López-Hernández (1994) y Pérez et al. (2005) como E. rigidum. Cruz-Hernández (2000) como E. anceps, además de registrar a E. cardiophorum.

Epidendrum chlorocorymbos Schltr., Repert. Spec. Nov. Regni Veg. Beih. 17: 30-31. 1922. (B, H, Ta, Te, Teno; SAP, SMSP, VS; Epífita; Cowan 3087, CAS, ENCB, NY; Noguera \& Cetzal 998, CH; Cowan \& Niño 3396, CSAT; Cowan et al. 3466, CSAT, ENCB, MEXU, MO, NY, UJAT, XAL; Ramón \& Sol 296, CSAT, UJAT; Cowan et al. 3396 CSAT, MO; Cruz \& Chamec 13, García 2, Magaña \& Ortíz 2388, UJAT). Referencias: (M, P) 1, 2, 4, 5*, 6, 7, 8*, 9, 10*, 11, 13*, Sánchez (2002: 570). Comentarios: Registrado por Cowan (1983) como E difforme Jacq. o E. ibaguense Kunth, ambos nombres fueron citados por Alderete (1992), además de incluir esta especie como E. chlorocorymbos. López-Hernández (1994) lo registró como Epidendrum difforme, E. aff. difforme y E. aff. chlorocorymbos. Los especímenes citados por Pérez et al. (2005) para E. chlorocorymbos, E. melistagum y E. radicans Pav. ex Lindl. corresponden a esta especie.

Epidendrum ciliare L., Syst. Nat. (ed. 10) 2: 1246. 1759. (H, Ta, Te; SAP, BM, SMSP; Epífita; Linden S.n., G; Linden 1215, F; Linden s.n., P; Cruz 2, Martínez 45, UJAT). Referencias: 1, 3*, 4, 8, 9, 10, 11, Sánchez y Hágsater (2010: 1311). ANP: 11.

Epidendrum cnemidophorum Lindl., Fol. Orchid. Epidendrum 53-54. 1853. (Te; SMSP; Epífita; Linden 1225, FI, W-R). Referencia: Sánchez y Hágsater (2010: 1322). Comentarios: Amenazada. 
Epidendrum cristatum Ruiz \& Pav., Syst. Veg. Fl. Peruv. Chil. 1: 243. 1798. Epidendrum raniferum Lindl., Gen. Sp. Orchid. Pl. 109. 1831. (Te; SMSP; Epífita; Linden s.n., AMES, P). Referencia: (Ta) 2, 4. Comentarios: La colección de Linden s.n. fue determinada como E. puctatum A.Richard \& Galeotti; sin embargo, esta representa un nomen nudum.

Epidendrum flexuosum G. Mey., Prim. Fl. Esseq. 260. 1818. Epidendrum imatophyllum Lindl., Gen. Sp. Orchid. PI. $106-107$. 1831. (C, Jo, M, Ta, Te, Teno; SAP, SBI, SMSP, MR, VS; Epífita; Matuda 3179, AMES, CAS, K, LL, MEXU, MICH, US; Cetzal \& Noguera 941, CH; Guadarrama \& López 768, CSAT, UJAT, XAL; Guadarrama et al. 5315, Guadarrama \& Ortíz 95.5.97, Izquierdo 68, Madrigal 9, Magaña 1852, Ortíz 27, Ortíz et al. 2135, 2158, Ramos et al. 731, Zamudio 1423, UJAT; Zamudio 309, UJAT, XAL; Alderete 109, XAL). Referencia: 1, 2, 4, 5, 7, 8, 9, 11, 13*. ANP: 1, 11, 14. Comentarios: Registrado por Cowan (1983) y Alderete y Cappello (1988: 39) como E. imatophyllum Lindl., y citado posteriormente por Alderete (1992) y RamónJiménez (1992).

Epidendrum galeottianum A.Rich. \& Galeotti, Ann. Sci. Nat., Bot., sér. 3, 3: 21. 1845. (C, M, Te, Teno; SAP, SMSP, VA; Epífita; Magaña \& Cowan 2978, CAS, CSAT, NY; Guadarrama 4154, Ortíz 2162, MEXU, UJAT; Cruz \& Ortíz 26, Mares 70, Mendieta 2, UJAT; Alderete 154, XAL). Referencias: (Ta) 1, 2, 3*, 4, 5*, 7, 9, Sánchez y Hágsater (2010: 1335). ANP: 1, 11. Comentarios: Registrado por Cowan (1983) como E. ibaguense H.B.K. (sic); por Alderete y Cappello (1988: 37, 43) como E. anceps Jacq. y E. secundum Jacq., respectivamente; por Alderete (1992) como E. anceps, E. fuscatum Sm. y E. ibaguense; Soto (1988) y Pérez et al. (2005) como E. anceps; por Ramón-Jiménez (1992) como E. secundum.

Epidendrum isomerum Schltr., Repert. Spec. Nov. Regni Veg. 2(22): 132-133. 1906. (Te; SBI, SMSP; Epífita; Ramón et al. 276, CSAT, UJAT; Ramón 412, UJAT). Referencias: 5*, 7, 9, 11*. ANP: 11.

Epidendrum isthmi Schltr., Repert. Spec. Nov. Regni Veg. Beih., 17: 34. 1922. (Te; SBI, SMSP; Epífita; Ramón 419, AMO, Cruz 4, Carrillo \& Cadena 15, UJAT). Referencias: 5*, 7*, 9, 11, 13*. (Protección especial). ANP: 11.

Epidendrum martinezii L.Sánchez \& Carnevali, Harvard Pap. Bot. 5(2): 408-410, f. 3. 2001. (B; SMSP; Epífita; Matuda 3349, AMES, MEXU, MICH). Referencias: 1, 4, 11, Sánchez y Hágsater (2010: 1355). Comentarios: La colección de Matuda (3349) fue identificada erróneamente como E. polyanthum por Cowan (1983) y citado posteriormente por Alderete (1992) y Pérez et al. (2005).

Epidendrum nocturnum Jacq., Enum. Syst. PI. 29. 1760. (B, H, Ta, Te; SMSP, SBI; Epífita; Cowan et al. 3261, CAS, CICY, CSAT, ENCB, MEXU, MO, NY, XAL; Noguera \& Savelli 977, CH; Guadarrama et al. 1091, Zamudio 455, 999, UJAT; Menéndez et al. 297, Valdivia 2103, 2055, 2094, XAL). Referencias: (Teno) 2, 4, 5*, 7, 9, 11, 12, 13*, Cervantes et al. (2002: 578). ANP: 11.

Epidendrum pachyrachis Ames, Sched. Orch. 2: 32-33. 1923. (Te; SMSP, VS; Epífita; Ramón 392, Guadarrama \& Ortíz 95.5.82, Ortíz s.n., MEXU, UJAT; Sol et al. 1090, UJAT). Referencias: 5*, 7, 9, 11*. ANP: 11.

Epidendrum polyanthum Lindl., Gen. Sp. Orchid. PI. 106. 1831. (Te; SAP; Epífita; Guadarrama et al. 1063, UJAT). Referencias: $3^{*}, 7,9,12 *$. ANP: 11.

Epidendrum ramosum Jacq., Enum. Syst. PI., 29. 1760. (Ta; SAP, SMSP; Epífita; Cruz \& Chamec 14, UJAT). Referencia: 9.

Epidendrum stamfordianum Bateman, Orchid Mexico \& Guatemala t. 11. 1838. (B, C, H, Ta, Te, Teno; SAP, SBI, SMSP, VS; Epífita; Noguera \& Cetzal 991, CH; Magaña \& Suárez 698, AMO, CICY, CSAT, XAL; Magaña \& Zamudio 745, Ramón \& Sol 243, CSAT; Menéndez et al. 440, MO, XAL; García et al. 30, Sol 425, Magaña 1923, Ortíz 660, UJAT; Ortíz 1, Zamudio 213, UJAT, XAL; Alderete 163, Valdivia 2029, 2082, XAL). Referencias: (Cen, M) 1, 2, 3, 4, 5*, 6, 7, 8*, 9, 11, $13^{*}$. ANP: 11.

Erycina pusilla (L.) N.H.Williams \& M.W.Chase, Lindleyana 16(2): 136. 2001. Epidendrum pusillum L., Sp. Pl. (ed. 2) 2: 1352. 1763. Psygmorchis pusilla (L.) Dodson \& Dressler, Phytologia 24(4): 288. 1972. Oncidium pusillum (L.) Rchb.f., Ann. Bot. Syst. 6(5): 714. 1863. (H; SAP; Epífita; Soriano-Santos s.n., UJAT). Referencias: 1, 3*, 8*, 9, 11. Comentarios: Registrado por Cowan (1983) como O. iridifolium H.B.K. (sic); por Alderete (1992) como O. pusillum, O. iridifolium y P. pusilla.

Eulophia alta (L.) Fawc. \& Rendle, Fl. Jamaica 1: 112-113. 1910. Limodorum altum L., Syst. Nat. (ed. 12) 2: 594. 1767. (C; VS; Terrestre; Magaña et al. 1011, CSAT, UJAT). Referencias: 8*, 9, 11. 
Gongora leucochila Lem., Fl. Serres Jard. Eur. 1: 87, t. 37. 1845. (M, Ta, Te; SAP, SMSP; Epífita; Cruz \& Chamec 16, Mendieta 11, Suárez 312, UJAT). Referencias: 3, 7, 9, 11, Salazar (2002: 587). ANP: 1, 11.

Gongora unicolor Schltr., Repert. Spec. Nov. Regni Veg. Beih. 19: 299. 1923. (Ta, Te; SAP, SMSP; Epífita; Noguera \& Cetzal 938, CH; Cruz \& Chamec 15, Ramón \& Sol 298, UJAT). Referencias: (M) 2, 4, 5*, 7, 9, 10*, 11, 13*. Comentarios: Registrada por Alderete y Cappello (1988: 47) como G. quinquenencis Ruiz \& Pavón (sic); por Alderete (1992) como G. aff. quinquenencis (sic); y por Ramón-Jiménez (1992) como G. quinquenervis Ruiz \& Pav.

Habenaria monorrhiza (Sw.) Rchb.f., Ber. Deutsch. Bot. Ges. 3: 274. 1885. Orchis monorrhiza Sw., Prodr. 118. 1788. (H; VA; Terrestre; Guadarrama et al. 4107, MEXU, UJAT). Referencias: 9, 11.

Habenaria pringlei B. L. Rob., Proc. Amer. Acad. Arts. 27: 184. 1892. (C, Ce, Jo, M, N; VA; Terrestre; Cowan \& Magaña 2880, CSAT; Magaña \& Guadarrama 2231, XAL, UJAT; Guadarrama et al. 6400, Novelo \& Ramos 2152, 2243, 2301, 2589, 3090, 3611, UJAT). Referencias: 3*, 4, 8*, 9, 10*, 11, 12, 13*. ANP: 11. Comentarios: Registrado por Cowan (1983) como H. bractescens y citado posteriormente por Alderete (1992); por Soto (1988) y Espejo y López-Ferrari (1997) como H. bractescens Lindl.

Habenaria repens Nutt., Gen. N. Amer. PI. 2: 190. 1818. (B, Ce, Co, H, M, Te; SBI, VA; Terrestre; Cowan et al. 2565, CSAT; Novelo \& Ramos 2235, MO, UJAT; Cruz 11, Guadarrama \& Ortíz 95.5.52, Guadarrama et al. 4108, Sousa et al. 13480, UJAT). Referencias: 1, 3*, 4, 5, 8*, 9, 11, 12, 13*. Comentarios: Registrado por Cowan (1983) como H. jaliscana S.Watson y H. limosa (Lindl.) Hemsl., y citados por Alderete (1992); por Cruz-Hernández (2000) como H. cf. brevilabiata A.Rich \& Galeotti y H. jaliscana. ANP: 11.

Habenaria trifida Kunth, Nov. Gen. Sp. (quarto ed.) 1: 330. 1815. (H; VA; Terrestre; Cowan 1541, CSAT; Guadarrama et al. s.n., MO, UJAT). Referencias: 1, 4*, 9, 11. Comentarios: Registrado por Cowan (1983) como H. pauciflora (Lindl.) Rchb.f. y citado por Alderete (1992).

Heterotaxis sessilis (Sw.) F.Barros, Hoehnea 29(2): 11. 2002. Epidendrum sessile Sw., Prodr. 122. 1788. Maxillaria crassifolia (Lindl.) Rchb.f., Bonplandia 2(2): 16. 1854. Heterotaxis crassifolia Lindl., Bot. Reg. 12: t. 1028. 1826. (Ta, Te; SAP, SBI, SMSP; Epífita; Noguera \& Cetzal 1006, CH; Ramón \& Sol 289, CSAT; Guadarrama 4150, Mares 68, UJAT). Referencias: 4, 5*, 7*, 9, 11. ANP: 11 .

Ionopsis utricularioides (Sw.) Lindl., Coll. Bot. 8: t. 39, f. A. 1826. Epidendrum utricularioides Sw., Prodr. 122. 1788. (C, Cen, Cu, H, Te, Teno; SBI, SMSP, VS; Epífita; Matuda 3464, AMES; Tenorio 19397, CICY; Alderete 320, Magaña 1132, CSAT; Moreno \& Ramos 287, CSAT, UJAT; Ortíz et al. 2186, UJAT). Referencias: 1, 3*, 4, 5*, 7, 8*, 9, 11, 12, 13*. ANP: 2.

Isochilus carnosiflorus Lindl., Paxton's Mag. Bot. 11: 213. 1844. (H, M, Ta, Te; SAP, SMSP, SBI; Epífita; Noguera \& Cetzal 946 CH; Magaña \& Suárez 199 CICY; Mares 71 MEXU, UJAT; Cruz et al. 25, Mendieta 3, Moreno \& Castillo 321, Ruíz et al. 54, UJAT). Referencias: 2, 3, 5*, 6, 7, 9, 11, 12*. ANP: 1, 11. Comentarios: Registrado por Alderete y Cappello (1988: 48), Alderete (1992) y López-Hernández (1994) como I. major Schltdl. \& Cham.; y por Cruz-Hernández (2000) como I. carnosiflorus y I. major.

Isochilus latibracteatus A.Rich. \& Galeotti, Ann. Sci. Nat., Bot., sér. 3, 3: 22. 1845. (H, Te; SMSP; Epífita; Cruz 9, Hanan 447, UJAT). Referencias: 7, 9, 11*. ANP: 11.

Jacquiniella equitantifolia (Ames) Dressler, Taxon 15(6): 242. 1966. Epidendrum equitantifolium Ames, Sched. Orch. 4: 39. 1923. (Ta, Te; SMSP; Epífita; Mendieta 8, UJAT). Referencias: 7, 8, 11*. ANP: 11.

Jacquiniella teretifolia (Sw.) Dressler, Sci. Surv. Porto Rico \& Virgin Islands 6(3): 340. 1926. (H; BM, SAP; Epífita; Cruz S.n. 38, Guadarrama et al. 4097, UJAT). Referencias: Registrado por Alderete (1992) como J. globosa (Jacq.) Schltr., y posteriormente citado por López-Hernández (1994) y Cruz-Hernández (2000).

Laelia rubescens Lindl., Edwards's Bot. Reg. 26: t. 41. 1840. Sinonimia completa en Halbinger y Soto (1997). (B; SMSP; Epífita; Valdivia 2047, 2086, MO, XAL; Valdivia 1992, XAL). Referencias: Halbinger y Soto (1997).

Lophiaris cosymbephora (C.Morren) R.Jiménez \& Carnevali, Harvard Pap. Bot. 6(1): 284. 2001. Oncidium cosymbephorum C.Morren, Ann. Soc. Roy. Agric. Gand. 5: 333-334, t. 275. 1849. Trichocentrum cosymbephorum (C.Morren) R.Jiménez \& Carnevali, Icon. Orchid. (Mexico) 5-6: ix. 2003. (Te; SMSP; Epífita; Ortíz s.n., UJAT). Referencias: 7, 8*, 9, 11. 
Lophiaris lindenii (Brogniart) Braem, Schlechteriana 4(1-2): 19. 1993. Oncidium lindenii Brogniart, Hort. Universel 3: $372-373$. 1842. Trichocentrum lindenii (Brogniart) M.W.Chase \& N.H.Williams, Lindleyana 16(2): 137. 2001. Oncidium retemeyerianum Rchb.f., Bot. Zeitung (Berlin) 14: 513. 1856. (B, M, Ta; SAP, SMSP, VS; Epífita; Noguera \& Cetzal 1029, CH; Matuda 3230, 28561, MEXU; Cruz 35, Cruz et al. 29, Sol 545, UJAT). Referencias: 1, 3*, 4, 8*, 9, 11, 13*. Comentarios: Registrado por Alderete (1992) como O. lindenii y O. retemeyerianum.

Lophiaris lurida (Lindl.) Braem, Schlechteriana 4(1-2): 19. 1993. Oncidium luridum Lindl., Bot. Reg. 9: pl. 727. 1823. Trichocentrum luridum (Lindl.) M.W.Chase \& N.H.Williams, Lindleyana 16(2): 137. 2001. (B, H, M, Ta, Te; SAP, SBI, SMSP, VS; Epífita; Magaña \& Curiel 969, CICY, MEXU; Noguera \& Cetzal 1020, CH; Magaña et al. 1039, MEXU; García \& Ramírez 26, García et al. 36, Magaña \& Guadarrama 2375, Ortíz et al. 738, UJAT; Alderete 123, Valdivia 2077, 2085, XAL). Referencias: 3*, 4, 5, 7, 8, 9, 10, 11, 13*. ANP: 11. Comentarios: Registrado por Alderete (1992) como O. altissimum (Jacq.) Sw. y O. guttatum (L.) Rchb.f.

Lophiaris oerstedii (Rchb.f.) R.Jiménez, Carnevali \& Dressler, Harvard Pap. Bot. 5(2): 423. 2001. Oncidium oerstedii Rchb.f., Bonplandia (Hannover) 2(7): 91. 1854. Trichocentrum oerstedii (Rchb.f.) R.Jiménez \& Carnevali, Icon. Orchid. (Mexico) 5-6: ix. 2002. Sinonimia completa ver Cetzal-Ix y Balam (2012). (B, C, Ce, H, JM, Jo, Ta, Te, Teno; MR, SAP, SMSP, SBI, VS; Epífita; Magaña 211, AMO; Noguera \& Cetzal 971, CH; Matuda 2856, Ortíz et al. 2191, MEXU; Guadarrama \& Ortíz 5194, MEXU, UJAT; Alcudia 21, Cruz s.n., 3, Moreno \& García 393, Novelo \& Ramos 2802, Ortíz et al. 2194, UJAT). Referencias: (Co, J, M) 1, 2, 3* 4, 5, 7, 8, 9, 10, 11, 12, 13*. ANP: 11. Comentarios: Registrado por Cowan (1983), Alderete y Cappello (1988: 62), Soto (1988), Alderete (1992), Ramón-Jiménez (1992) y Hanan-Alipi (1997) como O. carthagenense (Jacq.) Sw.

Lophiaris teaboana R.Jiménez, Carnevali \& Tapia-Muñoz, Harvard Pap. Bot. 5(2): 423. 2001. Trichocentrum × teaboanum (R.Jiménez, Carnevali \& Tapia-Muñoz) R.Jiménez \& Carnevali, Icon. Orchid. (Mexico) 5-6: ix. 2003. (Te; SAP, SMSP; Epífita; Carnevali \& Moreno 5908, CICY; Carnevali 6279, 6561; CICY; 6280, AMES, AMO, CICY). Referencias: 13*, Balam et al. (2011).

Lycaste aromatica (Graham) Lindl., Edwards's Bot. Reg. 29: misc. 16. 1843. Maxillaria aromatica Graham, Edinburgh New Philos. J. 1: 173-174. 1826. Referencias: (Ta; SMSP; Epífita; Matuda 28544, MEXU). Referencias: 2, 3, 6, 9, 11. Comentarios: Registro basado en la fotografía de Alderete y Cappello (1988: 49), y posteriormente identificado por Alderete (1992) como L. consobrina Rchb.f., nombre también usado por Cruz-Hernández (2000).

Maxillariella elatior (Rchb.f.) M.A.Blanco \& Carnevali, Lankesteriana 7(3): 528. 2007. Maxillaria elatior Rchb.f., Ann. Bot. Syst. 6(4): 532-533. 1863. (H, Ta; BM, SAP; Epífita; Noguera \& Cetzal 947, CH; Cruz et al. 30, UJAT). Referencias: 2, 4, 5, 6, 7, 9, 11*. Comentarios: Registrado por Cruz-Hernández (2000) bajo M. elatior y M. nasuta Rchb.f.

Maxillariella tenuifolia (Lindl.) M.A.Blanco \& Carnevali, Lankesteriana 7(3): 530. 2007. Maxillaria tenuifolia Lindl., Edwards's Bot. Reg. 23: sub t. 1986. 1837. (B, C, H, Ta, Te; SAP, SMSP; Epífita; Noguera \& Cetzal 1004, CH; Guadarrama 4153, CICY, UJAT; Cowan et al. 3515, CSAT, MO; Ventura 20149, CSAT; Cruz \& Ortíz 1, Ortíz \& Guadarrama 73, Mendieta 1, Ramón \& Sol 307, Zamudio 831, UJAT; Guadarrama et al. 1018, UJAT, XAL; Valdivia 1985, 2043, XAL). Referencias: (Teno) 1, 2, 3*, 4, 5*, 6, 7, 8*, 9, 11, 13*. ANP: 11. Comentarios: Registrado por Cruz-Hernández (2000) como M. meleagris Lindl. y M. tenuifolia.

Maxillariella variabilis (Bateman ex Lindl.) M.A.Blanco \& Carnevali, Lankesteriana 7(3): 530. 2007. Maxillaria variabilis Bateman ex Lindl., Edwards's Bot. Reg. 23: sub t. 1986. 1837. (Ta; SAP; Epífita; Noguera \& Cetzal 976 CH). Referencias: 9*, 11*, 13*. Comentarios: Nuevo registro.

Mesadenella petenensis (L.O.Williams) Garay, Bot. Mus. Leafl. 28(4): 335. 1982. Spiranthes petenensis L.O.Williams, Phytologia 25(7): 460-461. 1973. Stenorrhynchos petenensis (L.O.Williams) Burns-Bal. \& E.W.Greenw., Orquídea (Mexico City), n.s., 10(1): 93. 1986. (M; SBSP; Terrestre; Zamudio \& Guadarrama 850 UJAT). Referencias: (Ta; SAP) 2, 3*, 8*, 9, $13 *$. ANP: 1. Comentarios: Registrado por Alderete y Cappello (1988: 68-69) como Spiranthes sp.

Mormolyca ringens (Lindl.) Schltr., Orchideen 436 (1914). Trigonidium ringens Lindl., Edwards's Bot. Reg. $26:$ misc. 57.1840. (Ta; SMSP; Epífita; Noguera \& Cetzal 924, CH). Referencias: (Teno) 2, 3*, 4, 6, 8*, 9, 11, 13*.

Myrmecophila brysiana (Lem.) G.C.Kenn., Orchid Digest 43(6): 210-211. 1979. Schomburgkia brysiana Lem., Jard. Fleur. 1: Misc. 34, f. 1-4. 1851. (H; SBI; Epífita; Magaña \& Zamudio 245, MEXU, MO). Referencia: 11, 13*. 
Myrmecophila christinae var. christinae Carnevali \& Gómez-Juárez, Harvard Pap. Bot. 5(2): 434-437, f. 11-12. 2001. (C, Ce, Te, Teno; SBSP, SBI; Epífita; de la Cruz s.n., Magaña 1851, Novelo \& Ramos 2803, 3104, Pérez-Martínez S.n., UJAT; Guadarrama et al. 1062, Jiménez s.n., UJAT, XAL). Referencias: 2, 7, 9, 13*. ANP: 11, 14.Comentarios: registrado por Alderete y Cappello (1988: 56) como Myrmecophila sp.; por Hanan-Alipi (1997) como Maxillaria tibicinis (sic); y por Cruz-Hernández (2000) bajo Myrmecophila campechana (sic) y M. tibicinis. Nuevo registro.

Myrmecophila tibicinis (Bateman) Rolfe, Orchid Rev. 25(291): 51. 1917. Epidendrum tibicinis Bateman, Edwards's Bot. Reg. 24: misc. 8. 1838. (C, Co, H, P; MA, SBSP, SBI; Epífita; Ortíz 2139, 2165 UJAT; Alderete 152, Magaña \& Curiel 245, 473, 972, XAL). Referencias: $1,2,3^{*}, 4,8^{*}, 9,10^{*}, 11,12^{*}, 13^{*}$.

Nemaconia striata (Lindl.) Van den Berg, Salazar \& Soto Arenas, Neodiversity 2: 8. 2007. Ponera striata Lindl., Edwards's Bot. Reg. 28: Misc. 18-19. 1842. (B, Ta, Te; SBI, SMSP; Epífita; Noguera \& Cetzal 1034, CH; Mendieta 14, UJAT; Mares 69, UJAT, XAL; Valdivia 1995, 2065, XAL). Referencias: 2, 5*, 7*, 8*, 9, 11, 12, Hágsater y Soto (2002: 646). ANP: 11. Comentarios: Registrado por Alderete y Cappello (1988: 66) como Scaphyglottis sp.

Nidema boothii (Lindl.) Schltr., Repert. Spec. Nov. Regni Veg. Beih. 17: 43. 1922. Maxillaria boothii Lindl., Edwardss Bot. Reg. 24: misc. 52-53. 1838. (B, H, P, Ta, Te; SAP, SBSP, SMSP, SBI, VS; Epífita; Cowan \& Magaña 3147, CICY, MO, XAL; Noguera \& Cetzal 1016, CH; Cowan et al. 3262, MO; Magaña \& Zamudio 404, Valdivia 2026, MO, XAL; Cruz 20, García 11, García et al. 21, 2440, Ramón 175, 347, Zamudio 1000, UJAT; Magaña 1717 UJAT, XAL; Zamudio 1000, XAL). Referencias: (M, Teno) $1,2,3^{*}, 4,5^{*}, 6,7,8^{*}, 9,10^{*}, 11,13^{*}$. ANP: 11

Notylia barkeri Lindl., Edwards's Bot. Reg. 24: misc. 90. 1838. (B, H, M, Ta, Te; SAP, SBSP, SMSP, VS; Epífita; Xolocotzi 21977 AMES; Noguera \& Cetzal 995, CH; Ortíz 8 MEXU, UJAT; Cruz 548, Cruz \& García 28, 47.2, García et al. 34, Hanan et al. 295, Laparra 30, Mendieta 12, UJAT; Valdivia 2035, XAL). Referencias: 1, 2, 3*, 4, 5*, 6, 7, 9, 10*, 11, 13*. ANP: 1, 11.

Oeceoclades maculata (Lindl.) Lindl., Gen. Sp. Orchid. Pl., 237-238. 1833. Agraecum maculatum Lindl., Coll. Bot. 3: pl. 15.1821. (Ta; SMSP; Terrestre; Noguera \& Cetzal 963, CH). Comentarios: Nativa de África Tropical. Naturalizada. Nuevo registro.

Oncidium sotoanum R.Jiménez \& Hágsater, Lankesteriana 9(3): 415-419, f. 4, 5. 2010. (H, Te; SAP, SMSP; Epífita; Guadarrama et al. 1018, MEXU, UJAT; Cruz et al. 40, Ramón 174, UJAT). Referencias: 5*, 7, 9, 11. Comentarios: Registrado en los estudios citados como O. ornithorhynchum Kunth.

Oncidium sphacelatum Lindl., Sert. Orchid. sub. t. 48. 1841. (B, Ta, Te; SAP, SMSP, VS; Epífita; Espejo et al. 943, CICY, MO, UJAT, XAL; Noguera \& Cetzal 1024, CH; García \& Ramírez 47, Guadarrama et al. 1062, Ramón 182, Ramón \& Sol 229, Zamudio 217, UJAT; Valdivia 2059, XAL). Referencias: 4, 5*, 7, 9, 11, 13*. ANP: 11.

Ornithocephalus inflexus Lindl., Ann. Nat. Hist., 4(26): 384. 1840. (M, Ta, Te; SMSP; Epífita; Noguera \& Cetzal 1044, CH; Cruz \& Ortíz 23, Magaña 1563, UJAT). Referencias: 3, 5, 7, 8*, 9, 11. ANP: 1, 11. Comentarios: Registrado por Ramón-Jiménez (1992) como O. inflexus y O. iridifolius Rchb.f., este último nombre también fue citado por Hanan-Alipi (1997). Cruz-Hernández (2000) registró esta especie como O. inflexus, O. iridifolius y O. versiforus (sic).

Platystele stenostachya (Rchb.f.) Garay, Caldasia 8(40): 520-521. 1962. Pleurothallis stenostachya Rchb.f., Linnaea 18: 399. 1844. (Te; SMSP, SBI; Epífita; Cruz 151, Ortíz \& Guadarrama 72, Magaña 2121, Ruíz et al. 69, UJAT). Referencias: 5*, 7*, 9, 11, $12 *$. ANPS: 11 .

Pleurothallis leucantha Schltr., Repert. Spec. Nov. Regni Veg. 10(254-256): 353. 1912. (H, Ta; SAP, SMSP; Epífita; Guadarrama et al. 4105, UJAT). Referencias: 9, 11.

Polystachya caracasana Rchb.f., Bonplandia 2: 15. 1854. Sinonimia completa ver Peraza-Flores et al. (2012). (Ta, Te, Teno; SAP, SMSP, VS; Epífita; Noguera \& Cetzal 943, CH; Rovirosa 299 NY, PH; Cruz et al. 7, Ramón 50, 165, Ramón \& Sol 205, V.K.T. s.n. (sic), UJAT; Valdivia 1989 XAL). Referencias: 1, 3*, 4, 7, 8*, 9, 11, 13*, Peraza et al. (2011). ANP: 11. Comentarios: Registrado por Cowan (1983) como P. nana Reichb.f. (sic); por Soto (1988) y Espejo y López-Ferrari (1998) como P. foliosa (Hook.) Rchb.f.; por Alderete (1992) como P. aff. foliosa y P. cerea Lindl.; por López-Hernández (1994) como P. aff. foliosa; por Hanan-Alipi (1997) como P. cerea; y por Cruz-Hernández (2000) y Pérez et al. (2005) como P. foliosa y P. nana Reich. f. (sic). 
Polystachya clavata Lindl., Edwards's Bot. Reg.28: misc. 61-62. 1842. (B, H; SMSP; Epífita; Magaña \& Zamudio 410, CICY; Valdivia 2083, XAL). Comentarios: Registrado por Cowan (1983) como Spiranthes sp. Nuevo registro.

Prescottia stachyodes (Sw.) Lindl., Edwards's Bot. Reg. 22: sub t. 1915. Cranichis stachyodes Sw., Prodr. 120. 1788. (Ta; SMSP; Terrestre; Noguera \& Cetzal 964, CH). Referencias: 11*. Comentarios: Nuevo registro.

Prosthechea boothiana (Lindl.) W.E.Higgins, Phytologia 82(5): 376. 1997. Epidendrum boothianum Lindl., Edwards's Bot. Reg. 24: misc. 5-6. 1838. (Cen, Co; MR; Epífita; Ramos \& Novelo 3126, MO, UJAT; Díaz 208, UJAT). Referencias: Díaz-Jiménez (2007). ANPS: 11.

Prosthechea cochleata (L.) W.E.Higgins, Phytologia 82(5): 377. 1997. Epidendrum cochleatum L., Sp. Pl. (ed. 2) 2: 1351. 1763. Encyclia cochleata L. Brittonia 13(3): 264. 1961. (B, C, H, M, Ta, Te, Teno; SAP, SBI, SMSP, VS; Epífita; Noguera \& Cetzal 1003, CH; Matuda 3303, CAS; Magaña \& Cowan 2988, CAS, CSAT; Moreno \& García 328, CSAT, UJAT; Novelo et al. 180, MO; Cruz 12, 33, Ortíz et al. 1019, 2164, 2169, Zamudio 771, UJAT; Valdivia 2100, XAL). Referencias: (EZ, J) 1, 2, 3*, 4, 5*, 7, 8, 9, 10*, 11, 13*. ANP: $2,11$.

Prosthechea livida (Lindl.) W.E.Higgins, Phytologia 82(5): 379. 1997. Epidendrum lividum Lindl., Edwards's Bot. Reg. 24: misc. 51. 1838. Encyclia livida (Lindl.) Dressler, Brittonia 13(3): 265. 1961. (B, C, H, Jo, Te; MR, SBI, SMSP, VA; Epífita; Ortiz 2159, CICY; Matuda 3163, CAS; Fernández et al. 1474, CSAT, MO; Guadarrama 4151, Ortíz et al. 2130, 2159, UJAT). Referencias: 1, 3*, 4, 8*, 10, 11, 13*. ANP: 3, 14. Comentarios: Registrado por Cowan (1983) como Epidendrum deamii Schltr.; y por Alderete (1992) y Cruz-Hernández (2000) como Encyclia lívida y E. deamii.

Prosthechea pygmaea (Hook.) W.E.Higgins, Phytologia 82(5): 380. 1997. Epidendrum pygmaeum Hook., Bot. Mag. 60: t. 3233. 1833. Encyclia pygmaea (Hook.) Dressler, Brittonia 13(3): 265. 1961. (H, Ta, Te; SAP, SMSP; Epífita; Noguera \& Cetzal 979, CH; Ramón 422, CSAT; Guadarrama et al. 4097, UJAT). Referencias: 5*, 7*, 9, 11. Comentarios: Registrado por Cruz-Hernández (2000) como E. pigmaea y E. pseudopygmae (Finet.) Dressler \& Pollard.

Prosthechea radiata (Lindl.) W.E.Higgins, Phytologia 82(5): 380. 1997. Epidendrum radiatum Lindl., Edwards's Bot. Reg. 27: misc. 58. 1841. Encyclia radiata Lindl., Brittonia 13(3): 265. 1961. (B, C, H, Te; SAP, SBI, SMSP, VS; Epífita; Magaña 983, CICY, CSAT, ENCB, MEXU, XAL; Ramón 352, CSAT; Magaña \& Curiel 202, CSAT, ENCB, MEXU, MO, UJAT; Fernández \& Zamudio 1061, CSAT, ENCB, G, MEXU, UJAT; Arcía 69, ENCB; Barlow 30/163, F, MEXU; Zarate 72, MEXU; Valdivia 1991, MO; Guadarrama et al. 585, Magaña s.n., Magaña \& Ortíz 2385, Rebolledo 68, Soriana-Santos 71, UJAT; Alderete 48, Novelo 104, Valdivia 2046, XAL). Referencias: 1, 2, 4, 5*, 7*, 8*, 9, 11, 13*, Soto (2008: 1072). ANP: 8. Comentarios: Registrado por Ramón-Jiménez (1992) como Encyclea onephlebia (sic); por Hanan-Alipi (1997) como E. chacaoensis (Rchb.f.) Dressler \& G.E.Pollard; por Cruz-Hernández (2000) como E. ionophlebia (Reich.f.) Dressler y E. radiata; y por Pérez et al. (1997) como $P$. chacaoensis (Rchb.f.) W.E.Higgins (basado en Hanan-Alipi 1997) y P. radiata.

Restrepiella ophiocephala (Lindl.) Garay \& Dunst., Venez. Orchid. III. 4: 266. 1966. Pleurothallis ophiocephala Lindl., Edwards's Bot. Reg. 24: Misc. 34. 1838. (Ta; SAP; Epífita; Cruz 18, UJAT). Referencias: (Te) 2, 4, 6, 9, 11.

Rhetinantha aciantha (Rchb.f.) M.A.Blanco, Lankesteriana 7(3): 534. 2007. Maxillaria aciantha Rchb.f., Bot. Zeitung (Berlin), 10(49): 858. 1852. (Ta; SMSP; Epífita; Noguera \& Cetzal 1005, CH). Referencias: 11*. Comentarios. Nuevo registro.

Rhetinantha friedrichsthalii (Rchb.f.) M.A.Blanco, Lankesteriana 7(3): 534. 2007. Maxillaria friedrichsthalii Rchb.f., Bot. Zeitung 10(49): 858. 1852. (Te; SMSP; Epífita; Mares 66, UJAT). Referencias: 5*, 7, 9, 11, 12*.

Sacoila lanceolata (Aubl.) Garay, Bot. Mus. Leafl. 28(4): 352. 80. Limodorum lanceolatum Aubl., Hist. PI. Guiane. 2: 821.1775. Spiranthes lanceolata (Aubl.) León, Contr. Ocas. Mus. Hist. Nat. Colegio "De La Salle" 8: 358. 1946. Stenorrhynchos lanceolatum (Aubl.) Rich. ex Spreng., Syst. Veg. 3: 710. 1826. Satyrium orchioides Sw., Prodr. [O. P. Swartz] 118. 1788. Spiranthes orchioides (Sw.) Rich., Hist. Phys. Cuba, Pl. Vasc. 11: 252. 1850. (H, Jo; SBI, SMSP, VS; Terrestre; Ramírez et al. 651, CICY; Ortíz et al. 5308, Zamudio \& Suárez 286, UJAT; Magaña \& Trejo 1975, UJAT, XAL). Referencias: 1, 3*, 4, 8*, 9, 11, 13*. Comentarios: Registrado por Cruz-Hernández (2000) como S. lanceolata y Stenorrhynchos aurantiacum (La Llave \& Lex.) Lindl.

Sarcoglottis assurgens (Rchb.f.) Schltr., Beih. Bot. Centralbl. 37, Ab. 2: 414. 1920. Spiranthes assurgens Rchb.f., Beitr. Orchid.-K. C. Amer. 66. 1866. Spiranthes acaulis var. assurgens (Rchb.f.) Correll, Lloydia 10: 209. 1947. (Teno; SBI; Terrestre; Guadarrama et al. 1228, UJAT). Comentarios: Espécimen determinado como Sarcoglotis acaulis (sic). Nuevo registro. 
ORChiDACEAE DE TABASCO

Apéndice 1. Continuación

Sarcoglottis sceptrodes (Rchb.f.) Schltr., Beih. Bot. Centralbl., Abt. 2., 37 (Part 2, Heft 3): 421. 1920. Spiranthes sceptrodes Rchb. f., Bonplandia 3(15/16): 214. 1855. (M, Ta; SAP, SMSP; Terrestre; Noguera \& Cetzal 930, CH; Cruz et al. 29, Magaña et al. 1210, UJAT, XAL). Referencias: 2, 3*, 4, 8*, 9, 11*, 13*. ANPS: 1. Comentarios: Registrado por Alderete y Cappello (1988: 7273) como Spiranthes acaulis (J.E.S.) C y M (sic).

Scaphyglottis fasciculata Hook., Icon. PI. 4: t. 317. 1841. (Ta, Te; SMSP; Epífita; Noguera \& Cetzal 1041, CH; Mendieta 6, UJAT). Referencias: 7, 9, 11.

Scaphyglottis lindeniana (A.Rich. \& Galeotti) L.O.Williams, Ann. Missouri Bot. Gard. 28: 423. 1941. (H; BM, SAP; Epífita; Cruz \& Ortíz 10, UJAT). Referencias: 9.

Sobralia decora Bateman, Orchid. Mexico \& Guatemala t. 26. 1841. (H, Te; SAP, SMSP; Terrestre; Cruz 39, Guadarrama et al. 4098, 5054, Ramón \& Jiménez 381-a, UJAT). Referencias: 2, 4, 5*, 7, 9, 11. Comentarios: Registrado por Cruz-Hernández (2000) como S. decora y S. macdougalli.

Specklinia brighamii (S.Watson) Pridgeon \& M.W.Chase, Lindleyana 16(4): 256. 2001. Pleurothallis brighamii S.Watson, Proc. Amer. Acad. Arts. 23(2): 285-286. 1888. (Cen, M, Ta; SAP, SMSP; Epífita; Cowan et al. 2975, CSAT, MO; Lundell 1158, CICY; Cruz 151, Cruz \& Ortíz 22, García 96, Guadarrama \& Magaña 609, López s.n., Zamudio 314, UJAT; Zamudio1465, UJAT, XAL; Alderete 58, XAL). Referencias: 1, 2, 3*, 8*, 11, 13*. ANPS: 1. Comentarios: Registrado por Cowan (1983), Soto (1988) como P. tribuloides (Sw.) Lindl.; por Alderete (1992), Espejo y López-Ferrari (1998), Cruz-Hernández (2000) y Pérez et al. (2005) como P. brighamii y P. tribuloides.

Specklinia digitale (Luer) Pridgeon \& M.W.Chase, Lindleyana 16: 257. 2001. Pleurothallis digitale Luer, Orquídea (Mexico City), n.s. 6(1): 3-4, f. 1976. (Te; SMSP; Epífita; Martínez et al. 855, AMO). Referencia: 11, Solano (2008: 1091). Comentarios: Amenazada.

Specklinia grobyi (Bateman ex Lindl.) F.Barros, Hoehnea 10: 110. 1983. Pleurothallis grobyi Bateman ex Lindl. Edwards's Bot. Reg. 21: t. 1797. 1835. Specklinia grobyi (Bateman ex Lindl.) Pridgeon \& M.W.Chase, Lindleyana 16(4): 258. 2001. PleurothaIlis marginata Lindl., Edwards's Bot. Reg. 34: misc. 42. 1848. Specklinia marginata (Lindl.) Pridgeon \& M.W.Chase, Lindleyana 16(4): 258. 2001. (B, C, M, Ta, Te, Teno; SAP, SBI, SMSP; Epífita; Noguera \& Cetzal 950, CH; Cruz \& Ortíz 21, Guadarrama 4152, Guadarrama \& Magaña 607, Guadarrama \& Zuvieta 822, Jiménez 519, Ortíz et al. 2157, Ramón 56, Sol 545, Zamudio 315, UJAT). Referencias: 1, 3*, 4, 5*, 7, 8, 9, 10*, 11, 12*, 13*. Comentarios: Registrado por Cruz-Hernández (2000) como P. grobyi y P. marginata.

Specklinia pisinna (Luer) Solano \& Soto Arenas, Icon. Orchid. (Mexico) 5-6: xi. 2002. Pleurothallis pisinna Luer, Lindleyana 6(2): 105, f. 1991. (Te; SMSP; Epífita; Cruz 153, Mendieta 10, UJAT). Referencias: 7, 9, 11. ANP: 11.

Stanhopea oculata (G.Lodd.) Lindl., Gen. Sp. Orchid. PI. 158. 1832. (H; SAP; Epífita; Moreno \& García 330, UJAT). Comentarios: Nuevo registro.

Stelis ciliaris Lindl., Companion Bot. Mag. 2: 353. 1836. Apatostelis ciliaris (Lindl.) Garay, Bot. Mus. Leafl. 27(7-9): 188. 1979. (M, Ta; SMSP; Epífita; Cowan \& Niño 2984, CAS, CSAT, MEXU; Cowan \& Zamudio 3425, CAS, CSAT, MO). Referencias: 1, 4, $5,7,9,11,13 *$, Solano (1993).

Stelis cobanensis (Schltr.) Pridgeon \& M.W.Chase, Lindleyana 16(4): 262. 2001. Pleurothallis cobanensis Schltr., Repert. Spec. Nov. Regni Veg. 11: 42. 1912, (H; BM, SAP; Epífita; Cruz 31, Cruz \& Ortíz 5, Guadarrama et al. 4104, UJAT). Comentarios: Nuevo registro. Protección especial.

Stelis gracilis Ames, Orchidaceae 2: 266. 1908. (Ta; SMSP; Epífita; Cowan \& Zamudio 3425, CAS, CAST). Referencias: (P) 1, 4, 5, 8*, 9, 11, 13*, Solano (1993). Comentarios: Registrado por Ramón-Jiménez (1992) como S. aff. ovatilabia; por Hanan-Alipi (1997) como S. sp.; y por Cruz-Hernández (2000) como S. aff. ovatilabia y S. gracilis.

Trichosalpinx ciliaris (Lindl.) Luer, Phytologia 54(5): 395. 1983. Specklinia ciliaris Lindl., Edwards's Bot. Reg. 24: Misc. 31.1838. (B; SMSP; Epífita; Valdivia 2075, XAL). Referencias: 1, 3*, 8* 9*, 11*. Comentarios: Registrado por Cowan (1983) como Specklinia sp.; y Cruz-Hernández (2000) basado en Soto (1988). Nuevo registro. 
Apéndice 1. Continuación

Trigonidium egertonianum Bateman ex Lindl., Edwards's Bot. Reg. 24: misc. 73-74. 1838. (B, M, Ta, Te, Teno; SAP, SMSP; Epífita; Noguera \& Cetzal 1038, CH; Cruz 17, García et al. 39, Mendieta 4, Zamudio 311, UJAT; Alderete 126, Valdivia 493, 2060, XAL). Referencias: 2, 4, 5, 7, 9, 11. ANP: 11.

Vanilla inodora Schiede, Linnaea 4(4): 574-575. 1829. Vanilla pfaviana Rchb.f., Gard. Chron., n.s., 20: 230. 1883. (Te; SMSP; Hemiepífita; Ramón \& Sol 309 MEXU, UJAT). Referencias: 5*, 7*, 9, 11, Soto y Dressler (2010). Comentarios: Registrado por Cruz-Hernández (2000) como V. mexicana y V. planifolia.

Vanilla insignis Ames, Bot. Mus. Leafl. 2(8): 101-103. 1934. (B; SMSP; Hemiepífita; Valdivia 2063, XAL). Referencias: Soto (2002b: 700), Soto y Dressler (2010).

Vanilla odorata C.Presl, Reliq. Haenk. 1(2): 101. 1827. Vanilla ensifolia Rolfe, Bull. Misc. Inform. Kew 1892(65/66): 141. 1892. Epidendrum vermifugum Sessé \& Moc., Fl. Mexic. (ed. 2) 201. 1894. (C; SBI; Hemiepífita; Zamudio 825, UJAT). Referencias: 8*, 9, 10*, Soto y Dressler (2010).

Vanilla planifolia Andrews, Bot. Repos. 8: t. 538. 1808. Vanilla fragrans Ames, Sched. Orch. 7: 36. 1924. Sinonimia completa ver Soto y Dressler (2010). (C, N, Te, Teno; SAP, SBI, SMSP; Hemiepífita; Calónico et al. 21157, MEXU; Guadarrama et al. 618, Magaña 1010, Zamudio 825, UJAT; Márquez \& Guerrero 858, XAL). Referencias: 2, 4, 9, 11, Soto y Dressler (2010). Comentarios: Registrado por Cruz-Hernández (2000) como V. fragrans y V. planifolia. Protección especial. 